\title{
Molecular mechanism of m6A methylation of circDCL1 mediated by RNA methyltransferase METTL3 in the malignant proliferation of glioma cells
}

\section{Quansheng Wu}

Second Hospital of Shanxi Medical University

Xiaofeng Yin

Second Hospital of Shanxi Medical University

Wenbo Zhao

Second Hospital of Shanxi Medical University

Wenli Xu

Second Hospital of Shanxi Medical University

Laizhao Chen ( $\sim$ drchenlaizhao@163.com )

Second Hospital of Shanxi Medical University https://orcid.org/0000-0002-6964-9823

\section{Research Article}

Keywords: Glioma, Malignant proliferation, circDCL1, METTL3, m6A modification, miR-671-5p

Posted Date: January 25th, 2022

DOI: https://doi.org/10.21203/rs.3.rs-1233645/v1

License: (c) (i) This work is licensed under a Creative Commons Attribution 4.0 International License.

Read Full License 


\section{Abstract}

Glioma is an intracranial malignant tumor and remains largely incurable. circular RNAs are prominent modulators in glioma progression. This study investigated the function of circular RNA DLC1 (circDCL1) in the malignant proliferation of glioma cells. circDCL1 expression in glioma tissues and cells was determined using RT-qPCR. The effect of circDCL1 on the malignant proliferation of glioma cells was analyzed using CCK-8, colony formation, and EdU staining assays. METTL3, miR-671-5p, and CTNNBIP1 expressions were determined. $\mathrm{N}^{6}$ methyladenosine $\left(\mathrm{m}^{6} \mathrm{~A}\right)$ level of circDCL1 was analyzed using MeRIP. The binding relationship between miR-671-5p and circDCL1 or CTNNBIP1 was verified using RNA pulldown and dual-luciferase assays. A xenograft tumor model was established in nude mice to verify the effect of METTL3-mediated circDCL1 on glioma in vivo. circDCL1 was poorly expressed in glioma. circDCL1 overexpression suppressed glioma cell proliferation, while circDCL1 silencing showed an opposite trend. Mechanically, METTL3-mediated $\mathrm{m}^{6} \mathrm{~A}$ modification enhanced circDCL1 stability and upregulated circDCL1 expression in glioma. circDCL1 upregulated CTNNBIP1 transcription by competitively binding to miR-671-5p. METTL3 overexpression repressed the malignant proliferation of glioma via circDCL1/miR-671-5p/CTNNBIP1 in vivo. Collectively, METTL3-mediated $\mathrm{m}^{6} \mathrm{~A}$ modification upregulated circDCL1 expression, and circDCL1 promoted CTNNBIP1 transcription by sponging miR-671$5 p$, thus repressing the malignant proliferation of glioma.

\section{Introduction}

Glioma is a heterogeneous group of primary malignancies of the central nervous system, which is commonly graded I-IV according to histology and the degree of tumor invasion to surrounding tissues [1]. The most prevalent histological types of glioma in adults include astrocytomas, glioblastoma multiform, oligodendrogliomas, and mixed glioma [2]. Glioma is notorious for its rapid cell proliferation and angiogenesis, and particularly the tumors cells are resistant to conventional treatments, resulting in poor outcomes and a high incidence of relapse [3]. In addition to rapid proliferation, wide invasion, and drug resistance, the dismal outcomes of glioma patients also stem from the deficient knowledge of molecular pathogenesis and the lack of timely and effective diagnostic and therapeutic monitoring strategies [4]. Consequently, investigations on the precise molecular mechanism of glioma malignant proliferation and potent therapeutic targets have aroused extensive concerns.

circular RNAs (circRNAs), a novel class of covalently linked single-stranded RNAs with a closed loop structure, are primarily generated by back-splicing of transcription products [5]. Current evidence has unveiled that circRNAs are abundantly expressed in the brain tissues compared with other tissues, indicating their critical roles in various brain diseases [6]. circRNAs have differential expressions in glioma and participate in the modulation of glioma occurrence and development [7]. circDLC1 is derived from exons 14, 15, and 16 of the DLC1 gene, and circDLC1 overexpression is demonstrated to repress the proliferation of hepatoma cells [8]. Nevertheless, the exact role of circDLC1 in glioma remains unknown. 
Recently, $\mathrm{N}^{6}$-methyladenosine $\left(\mathrm{m}^{6} \mathrm{~A}\right)$ modification is emphasized for its abundant enrichment and critical biological functions in circRNAs [9]. $\mathrm{m}^{6} \mathrm{~A}$ modification emerges as methylation at the $\mathrm{N}^{6}$ position of adenosine, representing the most prevalent internal modification in eukaryotes mRNA [10]. The aberrant alternation of $\mathrm{m}^{6} \mathrm{~A}$ level affects RNA maturation, transcription, translation, and metabolism, which disrupts gene expression and vital cellular processes, and ultimately leads to the initiation and progression of multiple tumors, including glioma [11]. Methyltransferase-like 3 (METTL3) is identified as one of the "writers" for $\mathrm{m}^{6} \mathrm{~A}$ methylation that participate in regulating various cellular biological processes [12]. Notably, METTL3 is poorly expressed in glioma tissues, and METTL3 deficiency facilitates glioma cell proliferation [13]. METTL3 is also implicated in the biogenesis and function of circRNAs by mediating $\mathrm{m}^{6} \mathrm{~A}$ modification [9]. However, the mechanism of METTL3-mediated $\mathrm{m}^{6} \mathrm{~A}$ modification on circDCL1 in glioma has not been investigated before.

Mechanistically, circRNAs can modulate the expression of genes implicated in tumorigenesis and progression by serving as a competitive endogenous RNA (ceRNA) of microRNA (miRNA) [14]. miRNAs are a class of small endogenous non-coding RNAs (18-25 nt in length) that modulate gene expression at the post-transcriptional level [15]. miRNA expression profile affects the functions of genes implicated in gliomagenesis, tumor growth, therapeutic response, and prognosis which is accepted as a prospective therapeutic tool against malignant glioma [16]. Therefore, this study probed into the effect of METTL3mediated $\mathrm{m}^{6} \mathrm{~A}$ methylation of circDCL1 on the malignant proliferation of glioma cells and the downstream ceRNA mechanism of circDCL1, hoping to shed light on glioma treatment.

\section{Materials And Methods}

\section{Ethics statement}

This study was approved by the Ethical Committee of The Second Hospital of Shanxi Medical University and in accordance with Helsinki Declaration. All the animal experiments were implemented based on the Guide for the Care and Use of Laboratory Animals [17].

\section{Clinical sample collection}

We collected the tumor tissues of 40 patients (at an average age of $54.35 \pm 9.52 ; 21$ males and 19 females) with primary glioma and corresponding adjacent tissues in The Second Hospital of Shanxi Medical University from June 2016 to June 2018 and stored in liquid nitrogen at $-80^{\circ} \mathrm{C}$. None patients received chemotherapy or radiotherapy before. The histological features of collected tissues were independently diagnosed by 2 pathologists.

\section{Cell culture}

Human glioma cell lines (T98G, LN229, A172, and LN18) and healthy glioma cell line (HEB) were obtained from American Type Culture Collection (Manassas, Virginia, USA) and cultured in RPMI-1640 medium (HyClone, South Logan, UT, USA) containing 10\% heat-inactivated fetal bovine serum (Invitrogen, 
Carlsbad, CA, USA), $100 \mathrm{U} / \mathrm{mL}$ penicillin, and $100 \mu \mathrm{g} / \mathrm{mL}$ streptomycin (Thermo Fisher Scientific, Waltham, MA, USA) at $37^{\circ} \mathrm{C}$ with $5 \% \mathrm{CO}_{2}$.

\section{Cell treatment}

Three siRNAs specifically targeting circDCL1, circDCL1 pcDNA 3.1, METTL3 pcDNA 3.1, three siRNAs specifically targeting catenin beta interacting protein 1 (CTNNBIP1), and their negative controls (NCs) were designed and synthesized by GenePharma (Shanghai, China). Finally, these vectors were transfected into A172 or LN229 cells using Lipofectamine 3000 (Thermo Fisher Scientific).

\section{Cell counting kit-8 (CCK-8) assay}

A172 and LN229 cells were seeded into 96-well plates (2000 cells/well) and cultured for $0,24,48$, and 72 h. Each well was added with $10 \mu \mathrm{L}$ CCK-8 reagent (Beyotime, Beijing, China) for 2-h incubation at $37^{\circ} \mathrm{C}$. The absorbance at the wavelength of $450 \mathrm{~nm}$ was measured using a microplate reader (Bio-Rad, Philadelphia, PA, USA).

\section{Colony formation assay}

A172 or LN229 cells (200-300 cells) were seeded into the petri dish in humidified air at $37^{\circ} \mathrm{C}$ with $5 \% \mathrm{CO}_{2}$ for $10 \mathrm{~d}$. After the removal of the complete medium, the cells were fixed with $4 \%$ paraformaldehyde and stained with $0.1 \%$ crystal violet for 20 min. Colony cells were counted under an optical microscope (Nikon, Tokyo, Japan).

\section{5-ethynyl-2'-deoxyuridine (EdU) staining}

A172 and LN229 cells were seeded into 96-well plates (20000 cells) and cultured in the medium (100 $\mu \mathrm{L} /$ well, ThermoFisher) containing $50 \mu \mathrm{M}$ EdU for $2 \mathrm{~h}\left(5 \% \mathrm{CO}_{2}, 37^{\circ} \mathrm{C}\right)$. EdU binding detection kit (Ribobio, Guangzhou, Guangdong, China) was used for proliferation evaluation. Briefly, the cells were treated with $4 \%$ paraformaldehyde for $30 \mathrm{~min}$ and stained with $100 \mu \mathrm{L}$ Apollo dye solution (Ribobio) for $30 \mathrm{~min}$. The nuclei were stained with DAPI (ThermoFisher) for $30 \mathrm{~min}$, followed by observation under the fluorescence microscope (Olympus, Tokyo, Japan). The final results are presented as a percentage.

\section{Actinomycin D treatment}

A172 or LN229 cells were seeded into 24 -well plates $\left(5 \times 10^{5}\right.$ cells/well). After $24 \mathrm{~h}$, the cells were treated with $1 \mu \mathrm{g} / \mathrm{mL}$ actinomycin $D$ for $0,4,8,12,24,36,48$, and $72 \mathrm{~h}$. The relative RNA expressions of circDCL1 and DLC1 were analyzed using reverse transcription quantitative polymerase chain reaction (RT-qPCR).

\section{RNase R treatment assay}

The total RNA of A172 and LN229 cells was extracted using TRIzol reagent (Invitrogen). Then, $3 \mu \mathrm{g}$ total RNA was incubated with $10 \mathrm{U}$ RNase R (20 U/ $\mu \mathrm{L}$, Epicentre, Madison, WI, USA) at $37^{\circ} \mathrm{C}$ for $30 \mathrm{~min}$ and at $75^{\circ} \mathrm{C}$ for 10 min to deactivate the RNase R, followed by RT-qPCR.

\section{$\mathrm{m}^{6} \mathrm{~A}$ level detection}


The m6A level in total RNA was measured using m6A RNA methylation quantitative kit (ab185912, Abcam Inc., Cambridge, MA, USA). Firstly, RNA was coated in the detection well at $37^{\circ} \mathrm{C}$ for $90 \mathrm{~min}$, then added with capture antibody $(50 \mu \mathrm{L})$, detection antibody $(50 \mu \mathrm{L})$, and enhancer solution $(50 \mu \mathrm{L})$ respectively, and incubated at room temperature for $60 \mathrm{~min}$. Finally, the chromogenic solution was added for signal detection and absorbance measurement. The m6A level was measured using the colorimetry method. The absorbance of each well at $450 \mathrm{~nm}$ was read and calculated according to the standard curve. The final results were displayed as relative values.

\section{$\mathrm{m}^{6} \mathrm{~A}$ RNA immunoprecipitation (MeRIP) assay}

MeRIP was performed using Magna MeRIP m6A kit (Millipore, Billerica, MA, USA) to detect the m6A level of circDCL1. Briefly, $3 \mu \mathrm{g}$ anti-m6A antibody (ab208577, Abcam) was incubated with protein A/G magnetic beads at $4^{\circ} \mathrm{C}$ overnight. Then, the conjugate was incubated with RNA in the IP buffer containing RNase inhibitor and protease inhibitor. RNA was isolated and detected using RT-qPCR.

\section{Nuclear/cytosol fractionation assay}

After rinsing with pre-cooled phosphate-buffered saline (PBS), $1 \times 10^{6} \mathrm{~A} 172$ or LN229 cells were resuspended in $1 \mathrm{~mL}$ RLN buffer (50 mM Tris- $\mathrm{HCl} \mathrm{pH} \mathrm{7.4,} 0.14 \mathrm{M} \mathrm{NaCl}, 1.5 \mathrm{mM} \mathrm{MgCl}$, 0.5\% IGEPAL CA630 , and $1 \mathrm{mM} \mathrm{DTT}$ ), incubated on ice for $5 \mathrm{~min}$, and then homogenized. The cytoplasm was the supernatant after centrifugation. The precipitate was resuspended in $1 \mathrm{~mL}$ RSB buffer $(0.25 \mathrm{M}$ sucrose, $10 \mathrm{mM}$ Tris- $\mathrm{HCl} \mathrm{pH7.4,} 10 \mathrm{mM} \mathrm{NaCl}, 3 \mathrm{mM} \mathrm{MgCl}, 1 \mathrm{mM} \mathrm{DTT}$, and $0.5 \mathrm{mM}$ phenylmethylsulfonyl fluoride), homogenized, and centrifuged to remove the residual cytoplasm. The preliminary nucleus was resuspended in $3 \mathrm{~mL} 2 \mathrm{M}$ RSB (2 M sucrose, $10 \mathrm{mM}$ Tris- $\mathrm{HCl}$ pH7.4, $10 \mathrm{mM} \mathrm{NaCl}, 3 \mathrm{mM} \mathrm{MgCl}, 1 \mathrm{mM}$ DTT, and $0.5 \mathrm{mM} \mathrm{PMSF}$ ) and centrifuged at $3000 \mathrm{~g}$ for $45 \mathrm{~min}$. The isolated circDCL1 was subjected to RT-qPCR analysis, with U6 and GAPDH as nuclear and cytoplasmic controls, respectively [18].

\section{Dual-luciferase assay}

The binding site of cirCDCL1 and miR-671-5p was predicted on the Starbase database, and the binding site of miR-671-5p and CTNNBIP1 was predicted on the Targetscan database. Wild-type (WT) circDCL1 or CTNNBIP1 containing miR-671-5p binding sequence was synthesized and inserted into the pmirGLO vector (Promega, Madison, WI, USA) and named circDCL1 WT or CTNNBIP1 WT. Mutant-type (MUT) circDCL1 or CTNNBIP1 containing miR-671-5p mutant binding sequence was synthesized and inserted into the pmirGLO vector, named circDCL1 MUT or CTNNBIP1 MUT. The constructed plasmids were cotransfected with miR-671-5p mimic or mimic NC into A172 and LN229 cells using Lipofectamine 3000, respectively. The luciferase activity was measured after $48 \mathrm{~h}$ using a luciferase detection kit (Promega).

\section{RNA pull-down assay}

RNA pull-down assay was performed to explore the interaction between miR-671-5p and circDCL1 or CTNNBIP1 in A172 and LN229 cells. miR-671-5p and miR-NC were biotinylated using the biotin RNA Labeling Mix (Roche, Indianapolis, IN, USA) and transcribed using T7/SP6 RN polymerase (Roche). Then, biotin-labeled miR-671-5p (bio-miR-671-5p) and bio-miR-NC were treated with RNase free DNase I 
(Promega) and RNeasy Mini kit (Qiagen, Redwood City, CA, USA). Next, M-280 streptavidin magnetic beads were incubated with bio-miR-671-5p or bio-miR-NC at room temperature for $4 \mathrm{~h}$, and then with A172 and LN229 cell lysates. The RNA binding to bio-miR-671-5p or bio-miR-NC was extracted with TRIzol reagent for RT-qPCR.

\section{Xenograft tumor experiments}

Male BALB/c nude mice (aged 6 weeks old; weighing $20 \mathrm{~g}$ ) purchased from Vital River Laboratory Animal Technology Co., Ltd (Beijing, China) [SYXK (Beijing) 2017-0033] were raised in a standard animal room and maintained under a 12-h light/dark cycle, with food and water provided ad libitum. LN229 cells were infected with the lentiviral overexpression vector of METTL3 (LV-oe-METTL3) or NC (LV-oe-NC) obtained from Genechem (Shanghai, China). Afterward, puromycin was used to screen the stably expressed cells. LN229 cells $\left(5 \times 10^{5}\right)$ were injected into the posterior flank of mice subcutaneously and tumor growth was observed. The tumor size was measured every 3 days and the tumor volume was calculated: volume $\left(\mathrm{mm}^{3}\right)=\left(\right.$ length $\times$ width $\left.^{2}\right) / 2$. The mice were sacrificed by intraperitoneal injection of $1 \%$ pentobarbital sodium $(150 \mathrm{mg} / \mathrm{kg}) 21$ days after injection. The tumors were removed and weighed. The tumor tissue was fixed with $4 \%$ paraformaldehyde and embedded in paraffin for immunohistochemistry. The expression of cytokines in tumor homogenate was detected using RT-qPCR.

\section{Immunohistochemistry}

The tumor tissue of mice was fixed with $4 \%$ paraformaldehyde, embedded in paraffin, and cut into $4 \mu \mathrm{m}$ sections. After dewaxing and dehydration, the expressions of METTL3 and Ki-67 were analyzed using immunohistochemistry. In short, the sections were incubated with METTL3 antibody (ab195352, Abcam) and Ki67 antibody (ab15580, Abcam) at $4^{\circ} \mathrm{C}$ overnight. After PBS washing, the sections were incubated with the secondary antibody (ab6721, Abcam) at $37^{\circ} \mathrm{C}$ for $20 \mathrm{~min}$. After 2,4-diaminobutyric acid development (10 $\mathrm{min}$ ) and hematoxylin counterstaining (3 $\mathrm{min})$, the sections were dehydrated with gradient alcohol, cleared with xylene, and sealed with neutral resin, following observation under the microscope. Five visual fields were randomly selected from the section, and the positive rate of each visual field was calculated [19].

\section{RT-qPCR}

Total RNA was extracted from glioma tissues, transplanted tumor tissues, and cells using the TRIzol reagent (Invitrogen) and $2 \mu \mathrm{g}$ RNA was reverse transcribed into cDNA using SuperScript RT kit (Invitrogen). RT-qPCR was performed using SYBR Premix ExTaq ${ }^{\text {TM }}$ (Takara, Kyoto, Japan) and Applied Biosystems 7300 system (Applied Biosystems, Inc., Carlsbad, CA, USA). PCR primers are shown in Table 1. The relative expression was calculated using the $2^{-\triangle \Delta C t}$ method [20], with GAPDH and U6 as internal references [21]. 
Table 1

PCR primer sequences

\begin{tabular}{|c|c|}
\hline Name & Sequences $\left(5^{\prime}-3^{\prime}\right)$ \\
\hline \multirow[t]{2}{*}{ circDLC1 } & F: TATGGTGAGGGTCATGAAGGA \\
\hline & R: ACCAAATTACGAAGTGGTCGC \\
\hline \multirow[t]{2}{*}{ METTL3 } & F: TGGGGGTATGAACGGGTAGA \\
\hline & R: TGGTTGAAGCCTTGGGGATT \\
\hline \multirow[t]{2}{*}{ DLC1 } & F: AGCTTTTCCAGGTTCCCGAG \\
\hline & R: AGGGGCTTCAGCTCTTGTTC \\
\hline \multirow[t]{2}{*}{ miR-671-5p } & F: ATGAGTAGGAAGCCCTGGAG \\
\hline & R: TCAACTGGTGTCGTGGAGTC \\
\hline \multirow[t]{2}{*}{ CTNNBIP1 } & F: TATGCAGGGGTGGTCAACAG \\
\hline & R: GACCTGGAAAACGCCATCAC \\
\hline \multirow[t]{2}{*}{ GAPDH } & F: ATGGTTTACATGTTCCAATATGA \\
\hline & R: TTACTCCTTGGAGGCCATGTGG \\
\hline \multirow[t]{2}{*}{ U6 } & F: TCGCTTCGGCAGCACATATACT \\
\hline & R: GCTTCACGAATTTGCGTGTCATC \\
\hline
\end{tabular}

\section{Western blot}

Total protein was extracted from glioma tissues and cells using radio-immunoprecipitation assay buffer. The extract was centrifuged at $4^{\circ} \mathrm{C}$ and $12000 \mathrm{~g}$ for $15 \mathrm{~min}$ to collect the supernatant and the protein concentration was determined using bicinchoninic acid kits (Beyotime). The protein was separated using $10 \%$ SDS-PAGE and transferred onto polyvinylidene fluoride membranes (Millipore). The membranes were blocked with $5 \%$ skim milk for $1 \mathrm{~h}$ and incubated with the primary antibodies METTL3 (ab195352, 1:1000, Abcam) and GAPDH (ab9485, 1:2000, Abcam) at $4^{\circ} \mathrm{C}$ overnight. Following tris-buffered saline-tween buffer washing, the membranes were incubated with the secondary antibody (ab6721, 1:2000, Abcam) at $37^{\circ} \mathrm{C}$ for $1 \mathrm{~h}$. NIH Image J (National Institutes of Health, Bethesda, Maryland, USA) was used to analyze the gray value, with GAPDH as internal reference.

\section{Bioinformatics analysis}

The downstream miRNAs of circDCL1 were predicted through the Starbase database (http://starbase.sysu.edu.cn/index.php) [22]. The downstream genes of miR-671-5p were predicted through the Starbase database, Targetscan database (http://www.targetscan.org/vert_71/) [23], RNA22 v2 database (https://cm.jefferson.edu/rna22/Precomputed/) [24], and miRDB database (http://mirdb.org/index.html) [25]. 


\section{Statistical analysis}

Data analysis and map plotting were performed using the SPSS 21.0 (IBM Corp., Armonk, NY, USA) and GraphPad Prism 8.0 (GraphPad Software Inc., San Diego, CA, USA). The data complied with the assumption of normality and homogeneity of variance. Data are expressed as mean \pm standard deviation. The $t$ test was adopted for comparisons between two groups. One-way or two-way analysis of variance (ANOVA) was employed for the comparisons among multiple groups, following Tukey's multiple comparison test. A value of $p<0.01$ indicated a significant difference.

\section{Results}

\section{circDCL1 was poorly expressed in glioma tissues and cells}

circRNA has become a research hotspot in the field of glioma molecular biology with the characteristics of high content and good stability [26]. circDCL1 is poorly expressed in hepatocellular carcinoma [8], but its role in glioma is unclear. Therefore, we detected circDCL1 expression in collected glioma tissues and adjacent tissues. The results exhibited that circDCL1 expression was reduced in glioma tissues $(p<0.01$, Fig. 1A). Then, circDCL1 expression in human glioma cell lines (T98G, LN229, A172, and LN18) and healthy glioma cell line (HEB) was detected, and circDCL1 was also poorly expressed in glioma cells ( $p<$ 0.01 , Fig. 1B). In addition, circDCL1 level had no significant change after actinomycin $D$ treatment, but linear DLC1 level was decreased notably after actinomycin D treatment ( $p<0.01$, Fig. $1 C)$, and RNase R treatment assay showed that circDCL1 expression did not change significantly, but linear DLC1 expression was reduced significantly after RNase R incubation, indicating that circDCL1 was more stable than linear DLC1 $(p<0.01$, Fig. 1D). Briefly, the above results demonstrated that circDCL1 was poorly expressed in glioma tissues and cells.

\section{Overexpression of circDCL1 suppressed malignant proliferation of glioma cells}

To explore the effect of circDCL1 on the malignant proliferation of glioma cells, we intervened in the expression of circDCL1. circDCL1 pcDNA 3.1 (pc-circDCL1) was transfected into LN229 cells with relatively low circDCL1 expression and circDCL1 expression in LN229 cells was successfully upregulated $(p<0.01$, Fig. 2A); circDCL1 siRNA (si-circDCL1) was transfected into A172 cells with relatively high circDCL1 expression and circDCL1 expression in A172 cells was successfully downregulated $(p<0.01$, Fig. 2A), and si-circDCL1\#1 with the best intervention efficiency was selected for subsequent detection. CCK-8 and colony formation assays showed that circDCL1 overexpression decreased glioma cell proliferation and circDCL1 silencing enhanced glioma cell proliferation ( $p<0.01$, Fig. 2B-C). EdU assay results further confirmed this conclusion ( $p<0.01$, Fig. 2D). Briefly, overexpression of circDCL1 suppressed the malignant proliferation of glioma cells. 


\section{METTL3-mediated $\mathrm{m}^{6} \mathrm{~A}$ modification enhances circDCL1 stability and upregulates circDCL1 expression}

$\mathrm{m}^{6} \mathrm{~A}$ RNA methylation greatly affects RNA metabolism and plays a key role in cancer through a variety of mechanisms $[27,28] . \mathrm{m}^{6} \mathrm{~A}$ modification exists in circDCL1 [8]. METTL3, as an $\mathrm{m}^{6} \mathrm{~A}$ modifying enzyme, is poorly expressed in glioma $[28,13]$. We speculated that METTL3 regulated circDCL1 expression through $\mathrm{m}^{6} \mathrm{~A}$ modification. RT-qPCR results exhibited that METTL3 expression was reduced in glioma tissues and cells $\left(p<0.01\right.$, Fig. 3A-B), and Western blot results confirmed the same trend $\left(p<0.05\right.$, Fig. 3C-D). $\mathrm{m}^{6} \mathrm{~A}$ quantitative analysis showed that the $\mathrm{m}^{6} \mathrm{~A}$ level in glioma tissues and cells was consistent with the trend of METTL3 expression ( $p<0.01$, Fig. 3E-F). MeRIP showed that the $\mathrm{m}^{6} \mathrm{~A}$ level of circDCL1 was decreased in glioma tissues and cells $(p<0.01$, Fig. 3G-H). In 40 cases of glioma tissues, METTL3 expression was positively correlated with circDCL1 expression $(p<0.01$, Fig. 3I). To verify the regulatory effect of METTL3 on circDCL1, we transfected METTL3 pcDNA 3.1 (pc-METTL3) into glioma cells and METTL3 expression in cells was elevated significantly $(p<0.05$, Fig. 3J-K). With the increase of METTL3 expression, $\mathrm{m}^{6} \mathrm{~A}$ level in cells, circDCL1 expression, and $\mathrm{m}^{6} \mathrm{~A}$ level of circDCL1 were also increased $(p<$ 0.01 , Fig. $3 L-N$ ). In addition, the half-life of circDCL1 was prolonged after overexpression of METTL3 ( $p<$ 0.01 , Fig. 30). Briefly, METTL3-mediated $\mathrm{m}^{6} \mathrm{~A}$ modification upregulated circDCL1 expression by increasing the stability of circDCL1.

\section{Overexpression of METTL3 annulled the promoting effect of circDCL1 silencing on the malignant proliferation of glioma cells}

To verify the role of METTL3 in the regulation of malignant proliferation of glioma cells by circDCL1, we designed a combined experiment: A172 cells were treated with METTL3 overexpression and circDCL1 silencing. Compared with circDCL1 silencing alone, the combined treatment of METTL3 overexpression + circDCL1 silencing increased circDCL1 expression in glioma cells $(p<0.01$, Fig. 4A) and decreased glioma cell proliferation ( $p<0.01$, Fig. 4B-C). EdU staining showed that compared with the si-circDCL $1+$ pc-NC group, the cells in the si-circDCL1 + pc-METTL3 group had enhanced proliferation $(p<0.01$, Fig. 4D). Briefly, overexpression of METTL3 annulled the promoting effect of circDCL1 silencing on the malignant proliferation of glioma cells.

\section{circDCL1 promoted CTNNBIP1 transcription by competitively binding to miR-671-5p}

Next, we further explored the downstream mechanism of circDCL1. Firstly, nuclear/cytosol fractionation assay demonstrated that circDCL1 was mainly localized in the cytoplasm of glioma cells (Fig. 5A). Then, the downstream miRNAs of circDCL1 were predicted, in which miR-671-5p was highly expressed in glioma [29, 30]. The target genes of miR-671-5p were predicted and intersected (Fig. 5B), in which 
CTNNBIP1 was poorly expressed in glioma [31, 32]. Therefore, we speculated that the miR-671$5 p / C T N N B I P 1$ axis was a potential downstream mechanism of circDCL1. To verify our hypothesis, we identified the binding sites between circDCL1 and miR-671-5p, and miR-671-5p and CTNNBIP1 through the database (Fig. $5 \mathrm{C}$ ), and designed a dual-luciferase assay. The results confirmed that there were binding relationships between circDCL1 and miR-671-5p, and miR-671-5p and CTNNBIP1 $(p<0.01$, Fig. 5D). Meanwhile, RNA pull-down assay further emphasized the binding relationship between miR-671-5p and circDCL 1 or CTNNBIP1 ( $p<0.01$, Fig. 5E). RT-qPCR results exhibited that miR-671-5p expression was elevated, while CTNNBIP1 expression was reduced in glioma tissues and cells ( $p<0.05$, Fig. 5F-G). circDCL1 overexpression decreased miR-671-5p expression and circDCL1 silencing increased miR-671-5p expression, while miR-671-5p expression was decreased after the combined treatment of METTL3 overexpression and circDCL1 silencing; the expression trend of CTNNBIP1 was opposite to that of miR$671-5 p(p<0.01$, Fig. $5 \mathrm{H})$. In 40 cases of glioma tissues, miR-671-5p expression was negatively correlated with circDCL1 expression, and CTNNBIP1 expression was positively correlated with circDCL1 expression $(p<0.01$, Fig. $5 \mathrm{I})$. Briefly, circDCL1 promoted CTNNBIP1 transcription by competitively binding to miR671-5p.

\section{CTNNBIP1 silencing attenuated the inhibitory effect of circDCL1 overexpression on malignant proliferation of glioma cells}

Then, we verified the regulatory effect of CTNNBIP1 on glioma cells. After three CTNNBIP1 siRNAs (siCTNNBIP1) were transfected into LN229 cells, CTNNBIP1 mRNA expression in LN229 cells was decreased $(p<0.01$, Fig. $6 \mathrm{~A})$. We chose si-CTNNBIP1\#2 and pc-circDCL1 to treat LN229 cells for a combined experiment. Our results showed that the combined treatment of si-CTNNBIP1\#2 and pccircDCL1 significantly promoted the proliferation of glioma cells $(p<0.05$, Fig. 6B-D). Briefly, CTNNBIP1 silencing attenuated the inhibitory effect of circDCL1 overexpression on the malignant proliferation of glioma cells.

\section{METTL3 suppressed the malignant proliferation of glioma cells via the circDCL1/miR-671-5p/CTNNBIP1 axis in vivo}

Finally, we verified the regulation of METTL3-mediated circDCL1 on the malignant proliferation of glioma cells in vivo. Overexpression of METTL3 suppressed the tumor growth, manifested as reduced tumor volume and weight ( $p<0.01$, Fig. 7A-B), and decreased positive rate of Ki67 ( $p<0.01$, Fig. 7C). Compared with the LV-oe-NC group, the LV-oe-METTL3 group showed increased METTL3 expression $(p<0.01$, Fig. 7D-E), elevated m6A level and circDCL1 expression ( $p<0.01$, Fig. 7F-G), reduced miR-671-5p expression $(p<0.01$, Fig. 7H), and enhanced CTNNBIP1 expression $(p<0.01$, Fig. 7l). Briefly, METTL3 suppressed the malignant proliferation of glioma cells via the circDCL1/miR-671-5p/CTNNBIP1 axis in vivo. 


\section{Discussion}

Glioma presents a challenging clinical situation that causes notable mortality and morbidity [1]. circRNAs make a significant contribution to the regulation of cell cycle, angiogenesis, metastasis, and drug resistance of glioma cells [33]. This study elucidated that METTL3-mediated circDCL1 promoted CTNNBIP1 transcription by sponging miR-671-5p, thus repressing the malignant proliferation of glioma cells (Fig. 8).

It has been revealed that patients with lower circDLC1 expression exhibit a more advanced hepatocellular carcinoma stage, indicating that circDLC1 is correlated with hepatocellular carcinoma progression [8]. Nevertheless, the role of circDLC1 in glioma remained unknown. Herein, we collected 40 pairs of glioma tissues and adjacent tissues and cultured human glioma cell lines and healthy glioma cell lines, and found that circDLC1 was poorly expressed in glioma tissues and cells. Unlike linear RNAs, the circular structure of circRNAs contributes to their stability in tissues and ensures their capacity to participate in various biological events [34]. Consistently, we found that circDLC1 level did not change notably after actinomycin D or RNase R treatment, but linear DLC1 level was reduced significantly, indicating that circDLC1 was more stable than linear DLC1. To investigate the effect of circDLC1 on the malignant proliferation of glioma cells, we intervened in circDLC1 expression in glioma cells. pc-circDLC1 was transfected into LN229 cells with relatively low circDLC1 expression and si-circDLC1 was transfected into A172 cells with relatively high circDLC1 expression. Our results exhibited that circDLC1 overexpression suppressed glioma cell proliferation, while circDLC1 silencing showed an opposite trend. Briefly, we were the first to demonstrate that circDLC1 expression was reduced in glioma tissues and cells, and circDLC1 overexpression suppressed the malignant proliferation of glioma cells.

The biogenesis and functions of circRNAs can be mediated by $\mathrm{m}^{6} \mathrm{~A}$ modification and $\mathrm{m}^{6} \mathrm{~A}$ mediatedcircRNAs are implicated in a variety of cellular and physiological processes, which may confer potential therapeutic targets for human malignancies [35]. circDLC1 has been identified as a circRNA regulated by $\mathrm{m}^{6} \mathrm{~A}$ modification in hepatocellular carcinoma pathogenesis [8]. The reduction of $\mathrm{m}^{6} \mathrm{~A}$ level in glioma tissues can be partially attributed to the decreased METTL3 level [28]. METTL3 is a well-studied RNA methyltransferase that executes an $\mathrm{m}^{6} \mathrm{~A}$-dependent modification of circRNAs implicated in carcinogenesis $[36,37]$. Therefore, we speculated that METTL3 regulated circDLC1 expression in glioma through $\mathrm{m}^{6} \mathrm{~A}$ modification. Our results exhibited that the $\mathrm{m}^{6} \mathrm{~A}$ level, METTL3 expression, and the $\mathrm{m}^{6} \mathrm{~A}$ level of circDLC1 were reduced in glioma tissues and cells. METTL3 expression showed a positive correlation with circDLC1 in glioma tissues. Then, we transfected METTL3 pcDNA 3.1 into glioma cells. With the increase of METTL3 expression, the $\mathrm{m}^{6} \mathrm{~A}$ level, circDLC1 expression, and the $\mathrm{m}^{6} \mathrm{~A}$ level of circDLC1 were elevated in glioma cells. Moreover, the upregulation of METTL3 prolonged the half-life period of circDLC1. Consensus $\mathrm{m}^{6} \mathrm{~A}$ motifs are abundant in circRNAs and METTL3 enhances $\mathrm{m}^{6} \mathrm{~A}$-driven RNA translation [38]. We demonstrated that METTL3-mediated $\mathrm{m}^{6} \mathrm{~A}$ modification increased circDCL1 expression by enhancing the stability of circDCL1. 
Thereafter, we designed a combined experiment by treating A172 cells with METTL3 overexpression and circDLC1 silencing. Our results exhibited that the combined treatment of METTL3 overexpression and circDLC1 silencing reduced glioma cell proliferation. METTL3 expression is dramatically downregulated in glioma tissues and METTL3 downregulation underlies a potential mechanism of glioma tumorigenesis [13]. METTL3 knockdown dramatically facilitates glioblastoma stem cell growth, self-renewal, and tumor progression [39]. Similarly, we showed that METTL3 overexpression annulled the promoting effect of circDCL1 silencing on the malignant proliferation of glioma cells.

Concerning the downstream regulatory mechanism of circRNAs, it has been confirmed that circRNAs modulate the expression of target genes by functioning as a miRNA sponge [14]. Our results confirmed that cirCDCL1 was mainly expressed in the cytoplasm. circDLC1 was predicted to have a binding site with miR-671-5p. miR-671-5p is encoded by a gene localized at 7q36.1, which is amplified in glioblastoma multiforme [30]. miR-671-5p is highly expressed in glioblastoma cells, and facilitates the proliferation and reduces the apoptosis of glioblastoma cells [29]. Subsequently, the target genes of miR-671-5p were predicted, among which we focused on CTNNBIP1. CTNNBIP1 is accepted as a tumor suppressor that regulates the $\beta$-catenin signaling and is demonstrated to be diminished in glioma [32]. Dual-luciferase and RNA pull-down assays verified the binding relationships between miR-671-5p and circDCL1 or CTNNBIP1. We demonstrated that miR-671-5p expression was elevated and CTNNBIP1 expression was declined in glioma tissues and cells. circDLC1 upregulation reduced miR-671-5p expression, while circDLC1 downregulation enhanced miR-671-5p expression. miR-671-5p expression was negatively correlated with circDCL1 expression, and CTNNBIP1 expression was positively correlated with circDCL1 expression. All these confirmed that circDCL1 promoted CTNNBIP1 transcription by competitively binding to $\mathrm{miR}-671-5 p$.

Furthermore, the regulatory role of CTNNBIP1 in glioma cells was verified. si-CTNNBIP1 was transfected into LN229 cells overexpressing circDCL1, and the results exhibited that the combined treatment of CTNNBIP1 silencing and circDCL1 overexpression promoted glioma cell proliferation. CTNNBIP1 downregulation is related to unfavorable prognosis, high histological grade, and advanced glioma progression [31]. Consistently, our results demonstrated that CTNNBIP1 silencing attenuated the inhibitory effect of circDCL1 overexpression on the malignant proliferation of glioma cells. Finally, we verified the regulation of METTL3-mediated circDCL1 on the malignant proliferation of glioma cells in vivo. The reduction of METTL3-mediated $\mathrm{m}^{6} \mathrm{~A}$ modification results in the in vivo growth of glioma cells [13]. Our results exhibited that METTL3 overexpression repressed tumor growth, elevated $\mathrm{m}^{6} \mathrm{~A}$ level, and circdlc1 expression, reduced miR-671-5p expression, and enhanced CTNNBIP1 expression. Briefly, METTL3 suppressed the malignant proliferation of glioma cells via the circDCL1/miR-671-5p/CTNNBIP1 axis in vivo.

\section{Conclusion}

To sum up, METTL3-mediated $\mathrm{m}^{6} \mathrm{~A}$ modification upregulated circDCL1 expression by enhancing the stability of circDCL1, thereby promoting the competitive binding of circDCL1 and miR-671-5p, facilitating 
the transcription of CTNNBIP1, and eventually repressing the malignant proliferation of glioma cells. This study first reported circDCL1 expression patterns in glioma and elucidated its effect on the malignant proliferation of glioma cells. However, this study only explored the role of the miR-671-5p/CTNNBIP1 axis downstream of circDCL1, and there are still many miRNAs downstream of circDCL1 to be explored. Whether circDCL 1 is also affected by other $\mathrm{m}^{6} \mathrm{~A}$ modifying enzymes needs to be further studied. In addition, we only detected the effect of the change of CTNNBIP1 transcription level on the malignant proliferation of glioma cells, and the effect of the change of CTNNBIP1 protein level is not clear. In the future, we will verify the effect of CTNNBIP1 protein level on the malignant proliferation of glioma cells and explore other possible mechanisms of cirCDCL1.

\section{Abbreviations}

\begin{tabular}{|ll|}
\hline Abbreviations & Full name \\
\hline circRNAs & circular RNAs \\
\hline $\mathrm{m}^{6} \mathrm{~A}$ & $\mathrm{~N}^{6}$-methyladenosine \\
\hline METTL3 & Methyltransferase-like 3 \\
\hline ceRNA & competitive endogenous RNA \\
\hline miRNAs & microRNAs \\
\hline CTNNBIP1 & catenin beta interacting protein 1 \\
\hline NC & negative control \\
\hline PBS & phosphate-buffered saline \\
\hline RT-qPCR & Reverse transcription quantitative polymerase chain reaction \\
\hline CCK-8 & cell counting kit-8 \\
\hline EdU & 5-ethynyl-2'-deoxyuridine \\
\hline MeRIP & m ${ }^{6}$ A RNA immunoprecipitation \\
\hline ANOVA & analysis of variance \\
\hline
\end{tabular}

\section{Declarations}

\section{Author Contribution}

Quansheng Wu and Laizhao Chen have given substantial contributions to the conception and the design of the manuscript, Xiaofeng Yin, Wenbo Zhao and Wenli Xu contributed to acquisition, analysis and interpretation of the data. All authors have participated to drafting the manuscript, Quansheng Wu revised it critically. All authors read and approved the final version of the manuscript. 


\section{Funding}

The authors declare that no funds, grants, or other support were received during the preparation of this manuscript.

\section{Availability of Data and Materials}

The datasets generated during and/or analysed during the current study are available from the corresponding author on reasonable request.

\section{Consent to Participate}

All patients had consented to participate.

\section{Consent for Publication}

The consent to publish this manuscript has been obtained from all authors.

\section{Conflict of Interest}

The authors have no relevant financial or non-financial interests to disclose.

\section{Informed Consent}

All patients had signed the written informed consent.

\section{Acknowledgements}

Not applicable.

\section{References}

1. Ludwig K, Kornblum HI (2017) Molecular markers in glioma. J Neurooncol 134 (3):505-512. doi:10.1007/s11060-017-2379-y

2. Ostrom QT, Bauchet L, Davis FG, Deltour I, Fisher JL, Langer CE, Pekmezci M, Schwartzbaum JA, Turner MC, Walsh KM, Wrensch MR, Barnholtz-Sloan JS (2014) The epidemiology of glioma in adults: a "state of the science" review. Neuro Oncol 16 (7):896-913. doi:10.1093/neuonc/nou087

3. Xu S, Tang L, Li X, Fan F, Liu Z (2020) Immunotherapy for glioma: Current management and future application. Cancer Lett 476:1-12. doi:10.1016/j.canlet.2020.02.002

4. Alifieris C, Trafalis DT (2015) Glioblastoma multiforme: Pathogenesis and treatment. Pharmacol Ther 152:63-82. doi:10.1016/j.pharmthera.2015.05.005

5. Chen LL (2016) The biogenesis and emerging roles of circular RNAs. Nat Rev Mol Cell Biol 17 (4):205-211. doi:10.1038/nrm.2015.32 
6. Rybak-Wolf A, Stottmeister C, Glazar P, Jens M, Pino N, Giusti S, Hanan M, Behm M, Bartok O, AshwalFluss R, Herzog M, Schreyer L, Papavasileiou P, Ivanov A, Ohman M, Refojo D, Kadener S, Rajewsky N (2015) Circular RNAs in the Mammalian Brain Are Highly Abundant, Conserved, and Dynamically Expressed. Mol Cell 58 (5):870-885. doi:10.1016/j.molcel.2015.03.027

7. Hao Z, Hu S, Liu Z, Song W, Zhao Y, Li M (2019) Circular RNAs: Functions and Prospects in Glioma. J Mol Neurosci 67 (1):72-81. doi:10.1007/s12031-018-1211-2

8. Liu H, Lan T, Li H, Xu L, Chen X, Liao H, Chen X, Du J, Cai Y, Wang J, Li X, Huang J, Yuan K, Zeng Y (2021) Circular RNA circDLC1 inhibits MMP1-mediated liver cancer progression via interaction with HuR. Theranostics 11 (3):1396-1411. doi:10.7150/thno.53227

9. Zhang L, Hou C, Chen C, Guo Y, Yuan W, Yin D, Liu J, Sun Z (2020) The role of N(6)-methyladenosine $(\mathrm{m}(6) \mathrm{A})$ modification in the regulation of circRNAs. Mol Cancer 19 (1):105. doi:10.1186/s12943-02001224-3

10. Liu ZX, Li LM, Sun HL, Liu SM (2018) Link Between m6A Modification and Cancers. Front Bioeng Biotechnol 6:89. doi:10.3389/fbioe.2018.00089

11. Zhang Y, Geng X, Li Q, Xu J, Tan Y, Xiao M, Song J, Liu F, Fang C, Wang H (2020) m6A modification in RNA: biogenesis, functions and roles in gliomas. J Exp Clin Cancer Res 39 (1):192. doi:10.1186/s13046-020-01706-8

12. Zeng C, Huang W, Li Y, Weng H (2020) Roles of METTL3 in cancer: mechanisms and therapeutic targeting. J Hematol Oncol 13 (1):117. doi:10.1186/s13045-020-00951-w

13. Ji JW, Zhang YD, Lai YJ, Huang CG (2020) Mettl3 regulates the proliferation, migration and invasion of glioma cells by inhibiting PI3K/Akt signaling pathway. Eur Rev Med Pharmacol Sci 24 (7):38183828. doi:10.26355/eurrev_202004_20848

14. Qi X, Zhang DH, Wu N, Xiao JH, Wang X, Ma W (2015) ceRNA in cancer: possible functions and clinical implications. J Med Genet 52 (10):710-718. doi:10.1136/jmedgenet-2015-103334

15. Carbonell T, Gomes AV (2020) MicroRNAs in the regulation of cellular redox status and its implications in myocardial ischemia-reperfusion injury. Redox Biol 36:101607. doi:10.1016/j.redox.2020.101607

16. Wang BC, Ma J (2015) Role of MicroRNAs in Malignant Glioma. Chin Med J (Engl) 128 (9):12381244. doi:10.4103/0366-6999.156141

17. (2011). In: th (ed) Guide for the Care and Use of Laboratory Animals. The National Academies Collection: Reports funded by National Institutes of Health. Washington (DC). doi:10.17226/12910

18. Wu J, Li R, Li L, Gu Y, Zhan H, Zhou C, Zhong C (2020) MYC-activated IncRNA HNF1A-AS1 overexpression facilitates glioma progression via cooperating with miR-32-5p/SOX4 axis. Cancer Med 9 (17):6387-6398. doi:10.1002/cam4.3186

19. Cheng R, Chen Y, Zhou H, Wang B, Du Q, Chen Y (2018) B7-H3 expression and its correlation with clinicopathologic features, angiogenesis, and prognosis in intrahepatic cholangiocarcinoma. APMIS 126 (5):396-402. doi:10.1111/apm.12837 
20. Livak KJ, Schmittgen TD (2001) Analysis of relative gene expression data using real-time quantitative PCR and the 2(-Delta Delta C(T)) Method. Methods 25 (4):402-408.

doi:10.1006/meth.2001.1262

21. Liu Z, Guo S, Sun H, Bai Y, Song Z, Liu X (2020) Circular RNA CircHIPK3 Elevates CCND2 Expression and Promotes Cell Proliferation and Invasion Through miR-124 in Glioma. Front Genet 11:1013. doi:10.3389/fgene.2020.01013

22. Li JH, Liu S, Zhou H, Qu LH, Yang JH (2014) starBase v2.0: decoding miRNA-ceRNA, miRNA-ncRNA and protein-RNA interaction networks from large-scale CLIP-Seq data. Nucleic Acids Res 42 (Database issue):D92-97. doi:10.1093/nar/gkt1248

23. Agarwal V, Bell GW, Nam JW, Bartel DP (2015) Predicting effective microRNA target sites in mammalian mRNAs. Elife 4. doi:10.7554/eLife.05005

24. Miranda KC, Huynh T, Tay Y, Ang YS, Tam WL, Thomson AM, Lim B, Rigoutsos I (2006) A patternbased method for the identification of MicroRNA binding sites and their corresponding heteroduplexes. Cell 126 (6):1203-1217. doi:10.1016/j.cell.2006.07.031

25. Chen $Y$, Wang $X$ (2020) miRDB: an online database for prediction of functional microRNA targets. Nucleic Acids Res 48 (D1):D127-D131. doi:10.1093/nar/gkz757

26. Zhang Y, Lin X, Geng X, Shi L, Li Q, Liu F, Fang C, Wang H (2020) Advances in circular RNAs and their role in glioma (Review). Int J Oncol 57 (1):67-79. doi:10.3892/ijo.2020.5049

27. Wang T, Kong S, Tao M, Ju S (2020) The potential role of RNA N6-methyladenosine in Cancer progression. Mol Cancer 19 (1):88. doi:10.1186/s12943-020-01204-7

28. Li F, Zhang C, Zhang G (2019) m6A RNA Methylation Controls Proliferation of Human Glioma Cells by Influencing Cell Apoptosis. Cytogenet Genome Res 159 (3):119-125. doi:10.1159/000499062

29. Li X, Diao H (2019) Circular RNA circ_0001946 acts as a competing endogenous RNA to inhibit glioblastoma progression by modulating miR-671-5p and CDR1. J Cell Physiol 234 (8):13807-13819. doi:10.1002/jcp.28061

30. Barbagallo D, Condorelli A, Ragusa M, Salito L, Sammito M, Banelli B, Caltabiano R, Barbagallo G, Zappala A, Battaglia R, Cirnigliaro M, Lanzafame S, Vasquez E, Parenti R, Cicirata F, Di Pietro C, Romani M, Purrello M (2016) Dysregulated miR-671-5p / CDR1-AS / CDR1 / VSNL1 axis is involved in glioblastoma multiforme. Oncotarget 7 (4):4746-4759. doi:10.18632/oncotarget.6621

31. Tong YQ, Liu B, Zheng HY, Gu J, Liu H, Li F, Tan BH, Hartman M, Song C, Li Y (2015) MiR-215, an activator of the CTNNBIP1/beta-catenin pathway, is a marker of poor prognosis in human glioma. Oncotarget 6 (28):25024-25033. doi:10.18632/oncotarget.4622

32. Guo M, Zhang X, Wang G, Sun J, Jiang Z, Khadarian K, Yu S, Zhao Y, Xie C, Zhang K, Zhu M, Shen H, Lin Z, Jiang C, Shen J, Zheng Y (2015) miR-603 promotes glioma cell growth via Wnt/beta-catenin pathway by inhibiting WIF1 and CTNNBIP1. Cancer Lett 360 (1):76-86. doi:10.1016/j.canlet.2015.02.003

33. Geng X, Zhang Y, Li Q, Xi W, Yu W, Shi L, Lin X, Sun S, Wang H (2020) Screening and functional prediction of differentially expressed circular RNAs in human glioma of different grades. Aging 
(Albany NY) 13 (2):1989-2014. doi:10.18632/aging.202192

34. Jiang Y, Zhou J, Zhao J, Zhang H, Li L, Li H, Chen L, Hu J, Zheng W, Jing Z (2020) The U2AF2 /circRNA ARF1/miR-342-3p/ISL2 feedback loop regulates angiogenesis in glioma stem cells. J Exp Clin Cancer Res 39 (1):182. doi:10.1186/s13046-020-01691-y

35. Di Timoteo G, Dattilo D, Centron-Broco A, Colantoni A, Guarnacci M, Rossi F, Incarnato D, Oliviero S, Fatica A, Morlando M, Bozzoni I (2020) Modulation of circRNA Metabolism by m(6)A Modification. Cell Rep 31 (6):107641. doi:10.1016/j.celrep.2020.107641

36. Yi YC, Chen XY, Zhang J, Zhu JS (2020) Novel insights into the interplay between m(6)A modification and noncoding RNAs in cancer. Mol Cancer 19 (1):121. doi:10.1186/s12943-020-01233-2

37. Lin S, Choe J, Du P, Triboulet R, Gregory RI (2016) The m(6)A Methyltransferase METTL3 Promotes Translation in Human Cancer Cells. Mol Cell 62 (3):335-345. doi:10.1016/j.molcel.2016.03.021

38. Yang $Y$, Fan X, Mao M, Song X, Wu P, Zhang Y, Jin Y, Yang Y, Chen LL, Wang Y, Wong CC, Xiao X, Wang Z (2017) Extensive translation of circular RNAs driven by N(6)-methyladenosine. Cell Res 27 (5):626-641. doi:10.1038/cr.2017.31

39. Cui Q, Shi H, Ye P, Li L, Qu Q, Sun G, Sun G, Lu Z, Huang Y, Yang CG, Riggs AD, He C, Shi Y (2017) m(6)A RNA Methylation Regulates the Self-Renewal and Tumorigenesis of Glioblastoma Stem Cells. Cell Rep 18 (11):2622-2634. doi:10.1016/j.celrep.2017.02.059

\section{Figures}


A

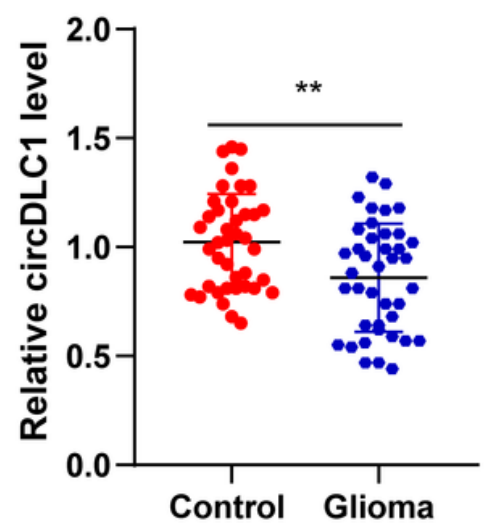

B

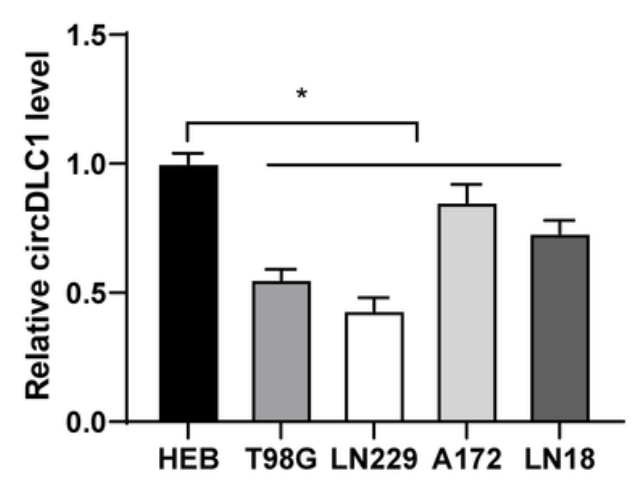

C

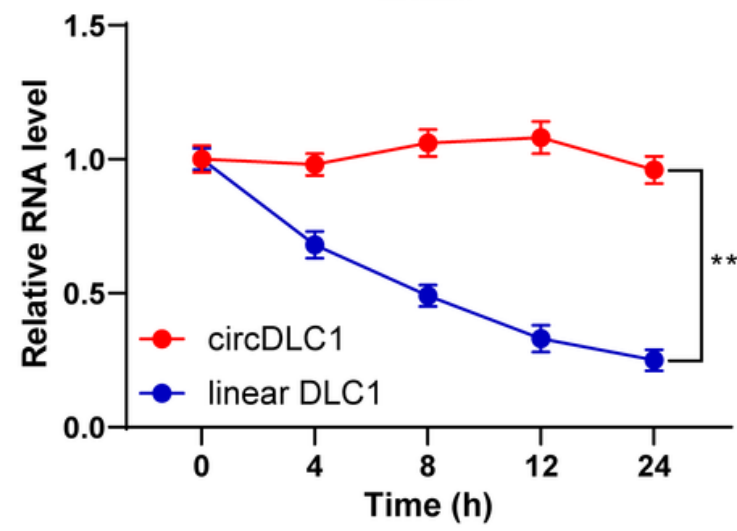

A172

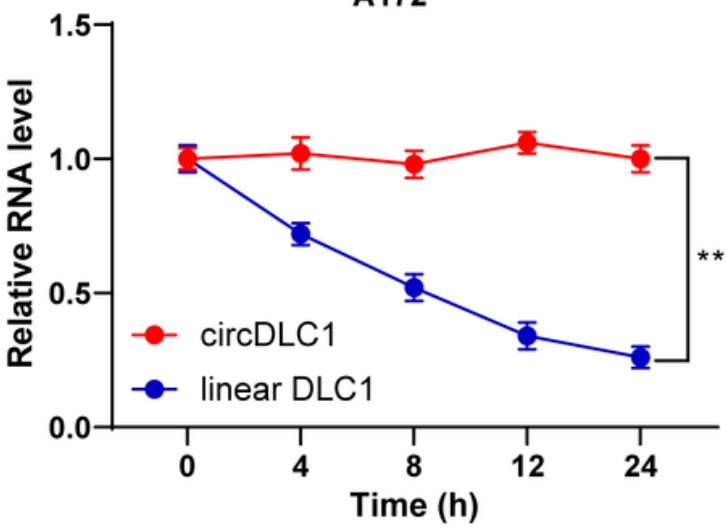

D

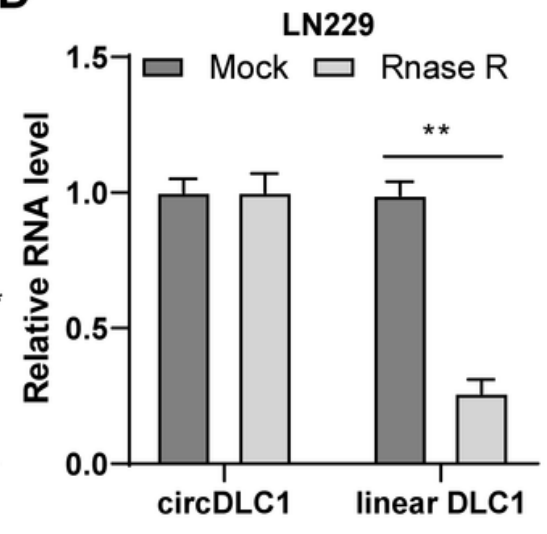

\section{Figure 1}

circDCL1 was poorly expressed in glioma tissues and cells. A: circDLC1 expression in 40 pairs of glioma tissues and adjacent tissues was determined using RT-qPCR. B: circDLC1 expression in human glioma cell lines and normal glioma cell line was detected using RT-qPCR. C: After actinomycin D treatment, the expressions of circDLC1 and linear DLC1 in cells were detected using RT-qPCR. D: After RNase R treatment, the expressions of circDLC1 and linear DLC1 in cells were detected using RT-qPCR. N $=40$. The cell experiment was repeated 3 times independently. Data in panels B-D are presented as mean \pm standard deviation. Data in panel A were analyzed using paired t-test. Data in panel B were analyzed using one-way ANOVA, and data in panels C-D were analyzed using two-way ANOVA, followed by Tukey's multiple comparisons test, ${ }^{*} p<0.05,{ }^{\star *} p<0.01$. 
A

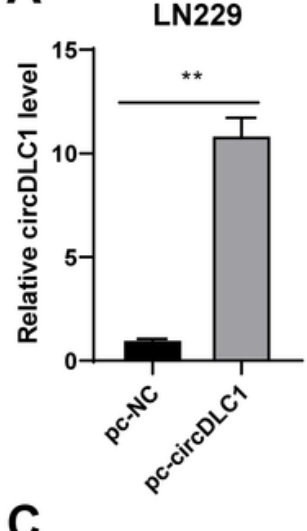

C

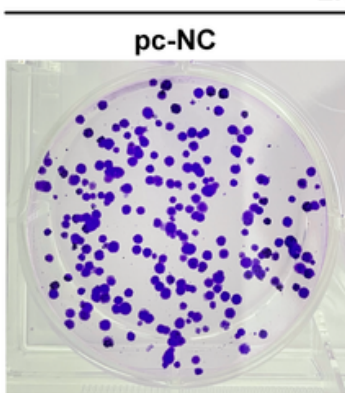

A172

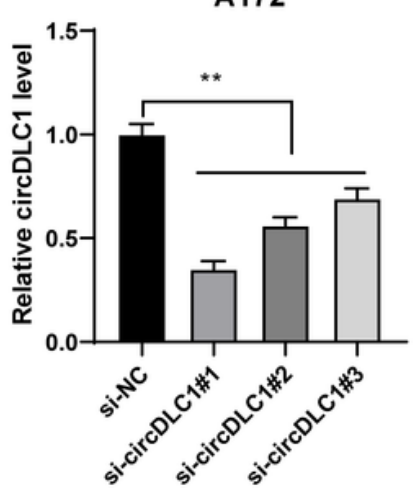

LN229

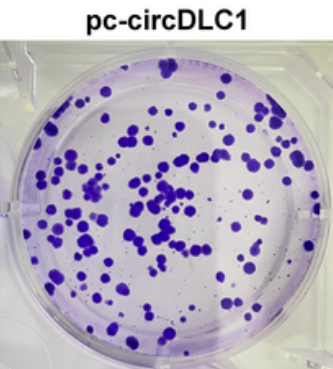

D

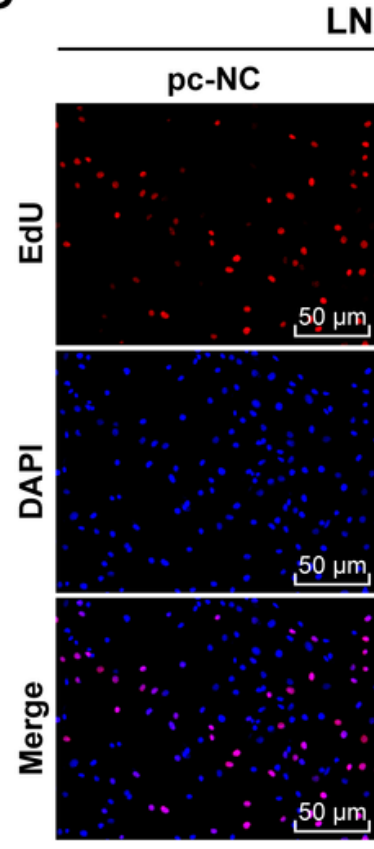

B
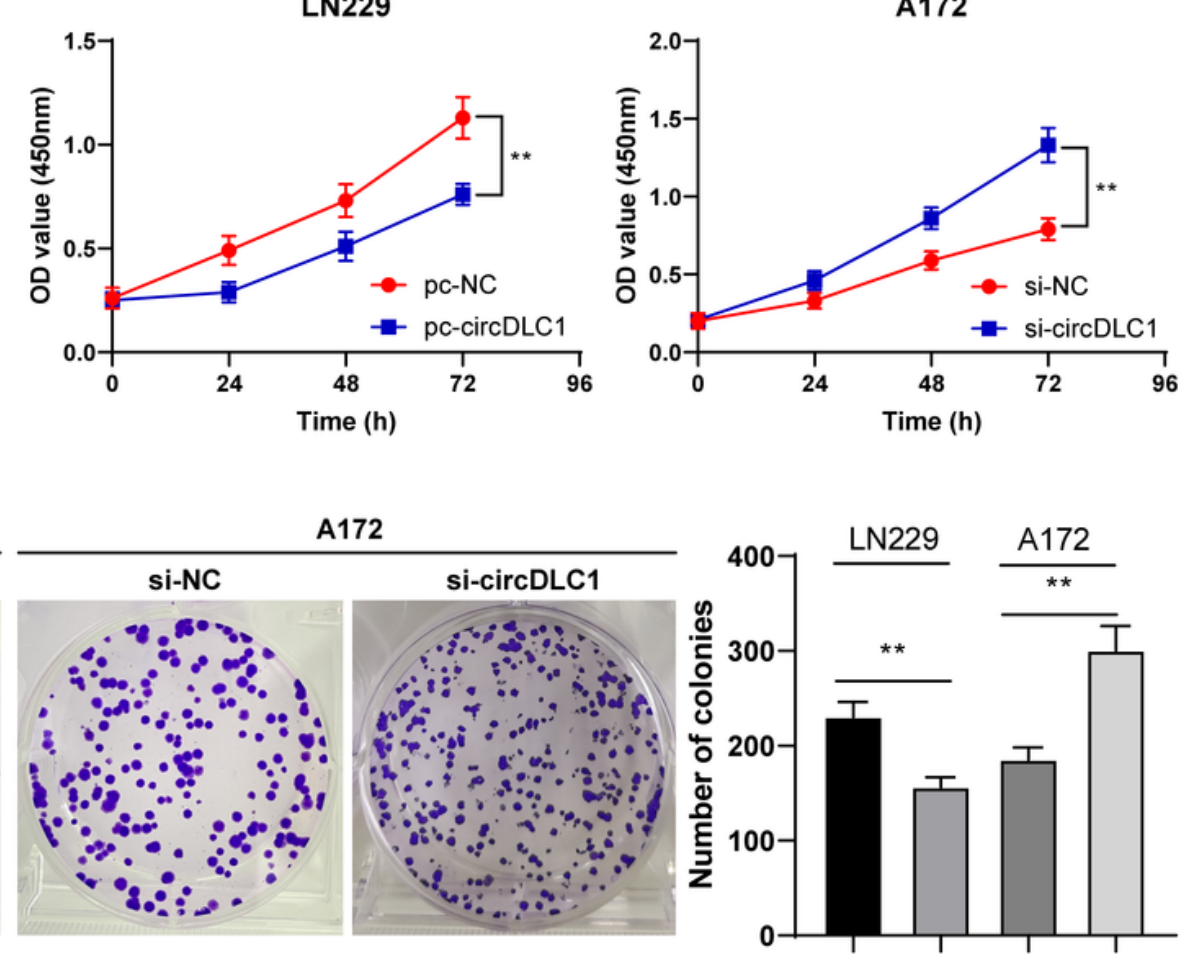

A172

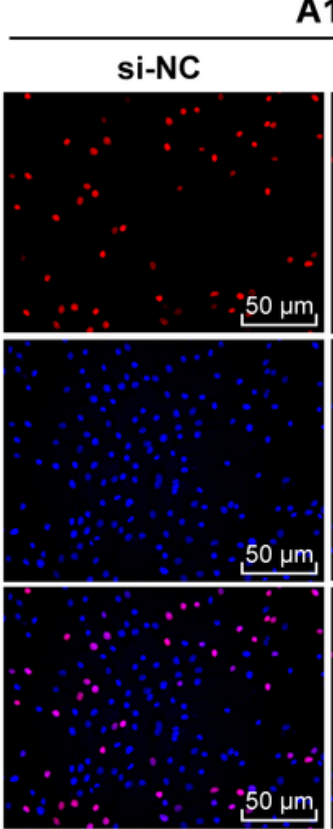

si-circDLC1
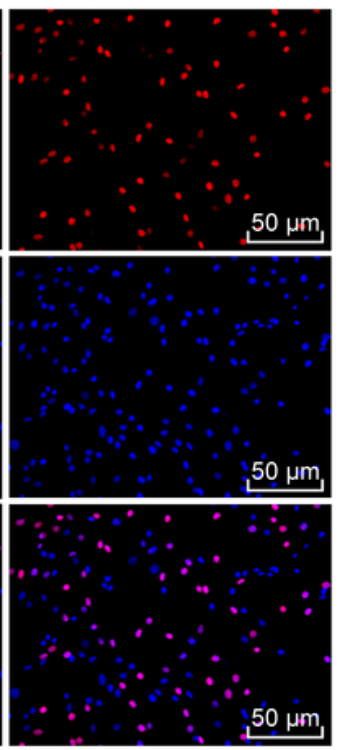

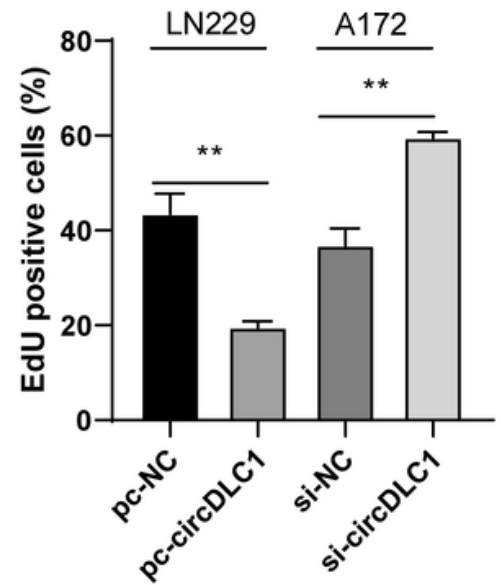

Figure 2

Overexpression of circDCL1 suppressed malignant proliferation of glioma cells. circDLC1 pcDNA 3.1 (pccircDLC1) was transfected into LN229 cells, with NC pcDNA 3.1 (pc-NC) as control, circDLC1 siRNA (sicircDLC1) was transfected into A172 cells, with NC siRNA (si-NC) as control. A: circDLC1 expression in cells was detected using RT-qPCR. B-D: Cell proliferation in each group was measured using CCK-8 assay (B), colony formation assay (C), and EdU staining (D). The cell experiment was repeated 3 times independently. Data are presented as mean \pm standard deviation. The comparisons between two groups 
in panels $\mathrm{A} / \mathrm{C}$-D were analyzed using t-test. The comparisons among multiple groups in panel A were analyzed using one-way ANOVA, and the comparisons among multiple groups in panel B were analyzed using two-way ANOVA, followed by Tukey's multiple comparisons test, ${ }^{* \star} p<0.01$.
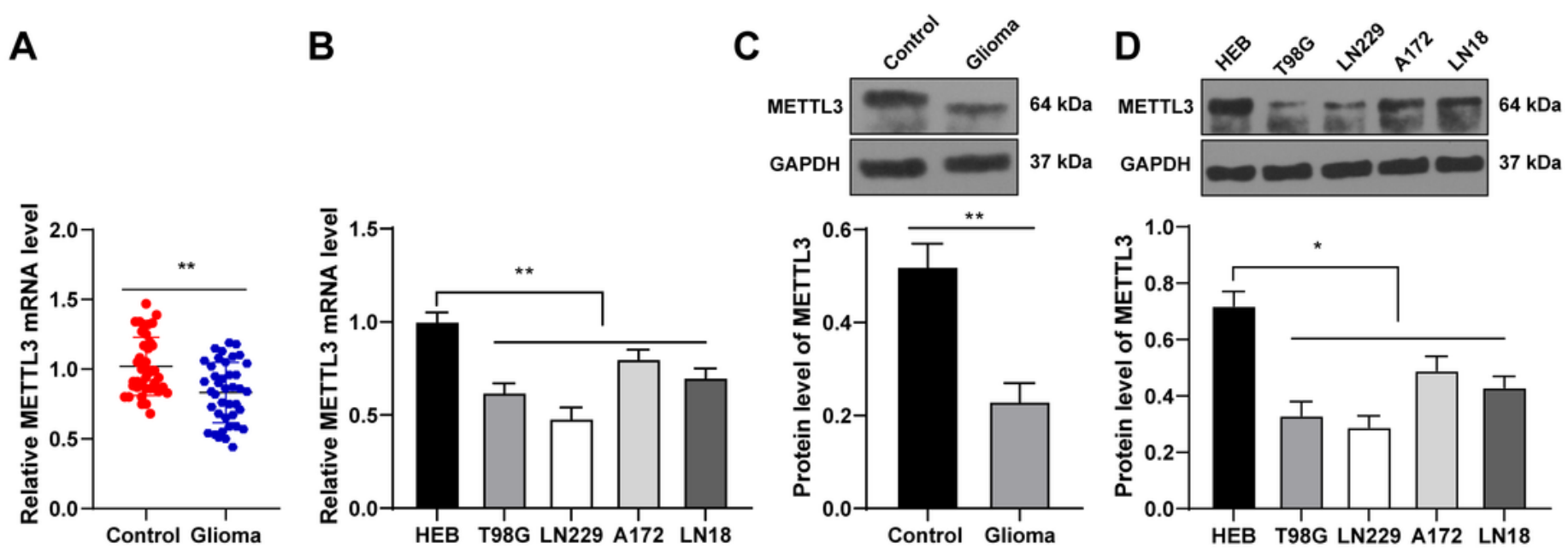

E

$\mathbf{F}$
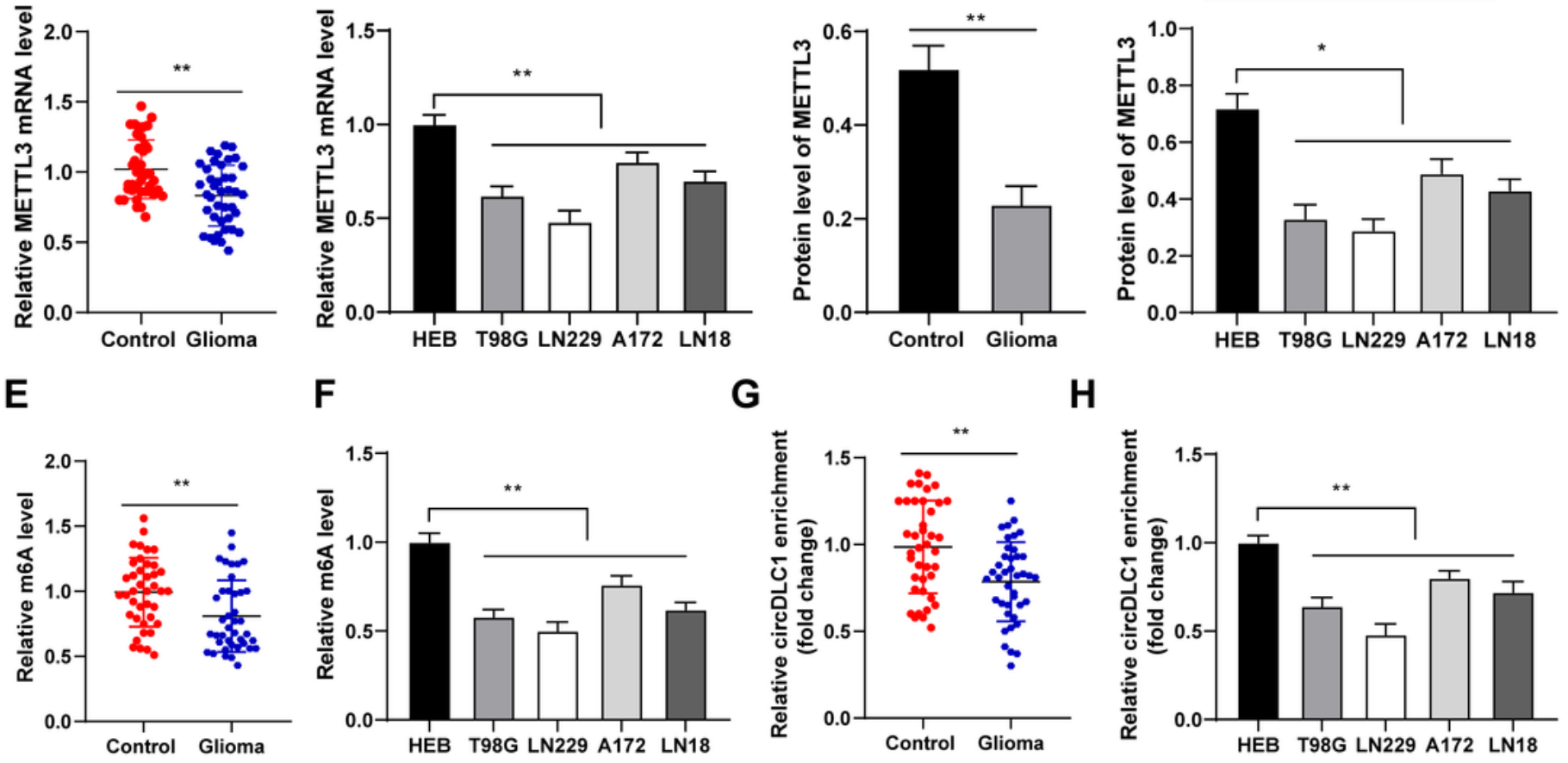

G
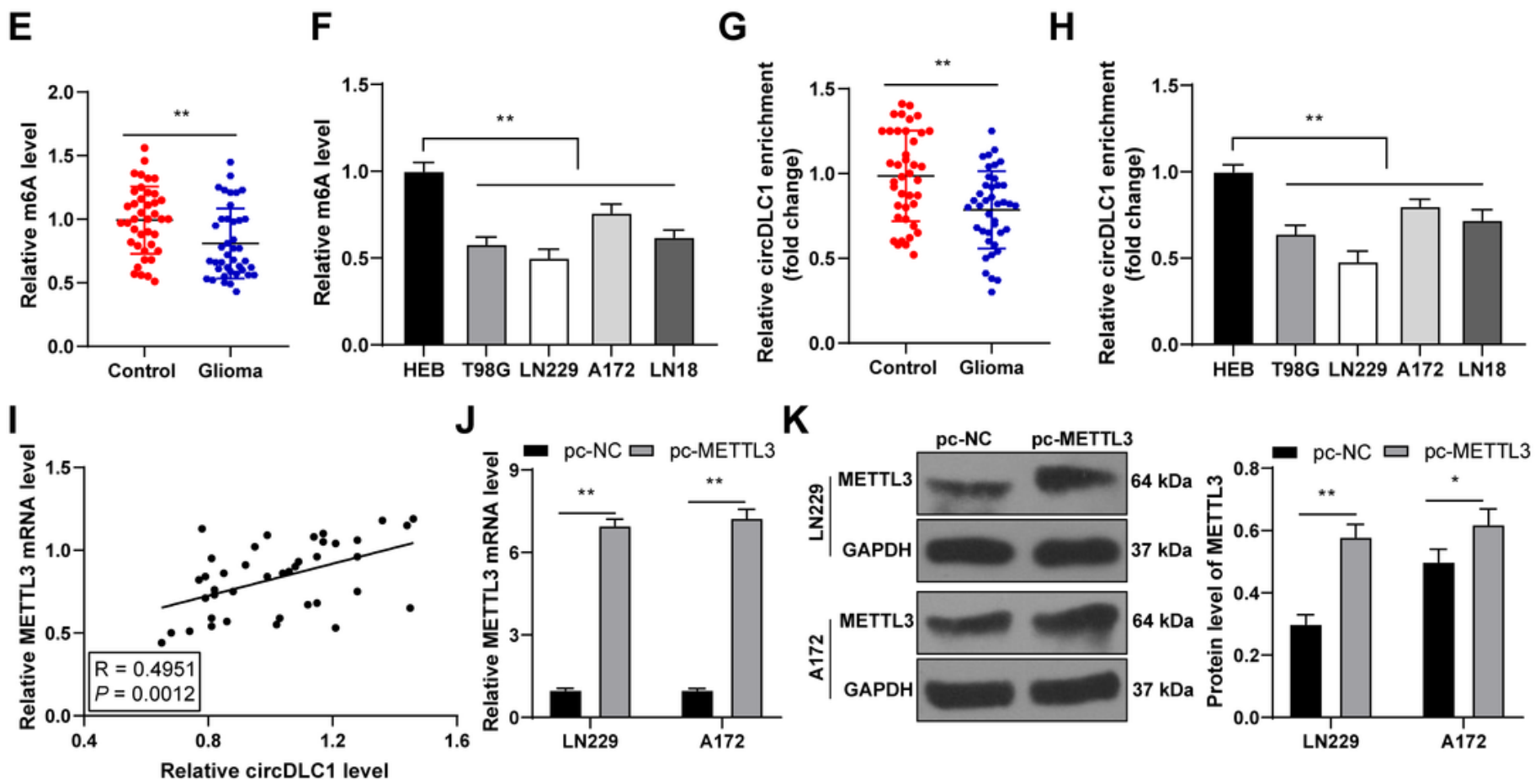

K
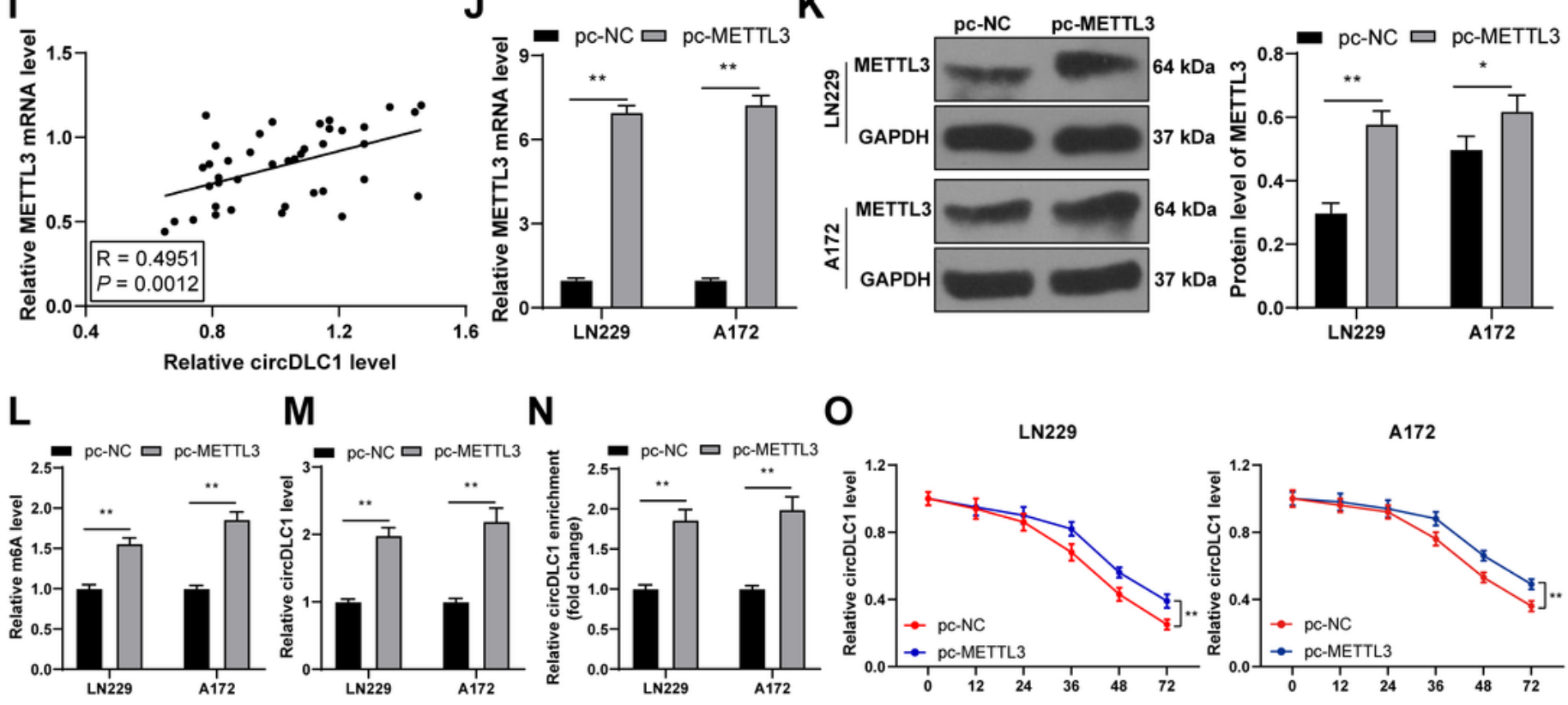

0
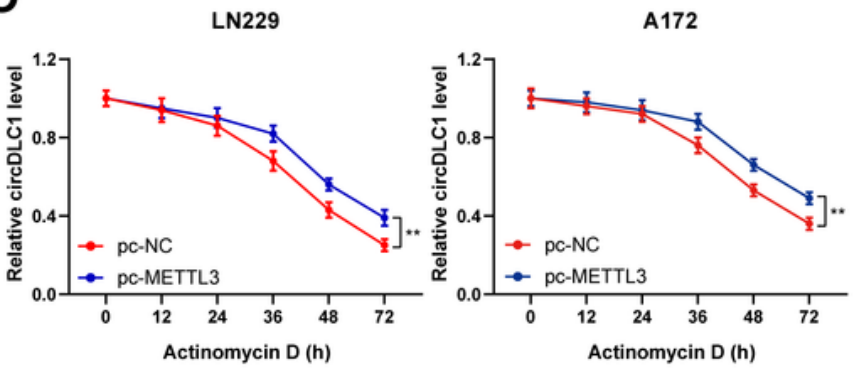

Figure 3 
METTL3-mediated $\mathrm{m}^{6} \mathrm{~A}$ modification enhances circDCL1 stability and upregulates circDCL1 expression. A-D: METTL3 expression in glioma tissues and cells was detected using RT-qPCR and Western blot. E-F: $\mathrm{m}^{6} \mathrm{~A}$ quantitative analysis of $\mathrm{m}^{6} \mathrm{~A}$ level in tumor tissues and cells. G-H: MeRIP analysis of $\mathrm{m}^{6} \mathrm{~A}$ level of circDCL1 in tumor tissues and cells. I: Pearson correlation analysis of the correlation between METTL3 and circDCL1 in 40 cases of glioma. METTL3 pcDNA 3.1 (pc-METTL3) was transfected into LN229 and A172 cells respectively, with NC pcDNA 3.1 (pc-NC) as NC. J-K: METTL3 expression in cells was detected using RT-qPCR and Western blot. L: $\mathrm{m}^{6} \mathrm{~A}$ quantitative analysis of $\mathrm{m}^{6} \mathrm{~A}$ level in cells. M: circDCL1 expression in cells was detected using RT-qPCR. N: MeRIP analysis of $\mathrm{m}^{6} \mathrm{~A}$ level of circDCL1 in cells. $\mathrm{O}$ : After actinomycin $D$ treatment, circDCL1 expression in cells was detected using RT-qPCR. $N=40$. The cell experiment was repeated 3 times independently. Data in panels B-D/F/H/J-O are presented as mean \pm standard deviation. The comparisons between two groups in panels $A / E / G$ were analyzed using paired ttest, and the comparisons between two groups in panels $\mathrm{C} / \mathrm{J}-\mathrm{N}$ were analyzed using t-test. The comparisons among multiple groups in panels $B / D / F / H$ were analyzed using one-way ANOVA, and the comparisons among multiple groups in panel $\mathrm{O}$ were analyzed using two-way ANOVA, followed by Tukey's multiple comparisons test, ${ }^{*} p<0.05,{ }^{* *} p<0.01$. 
A B

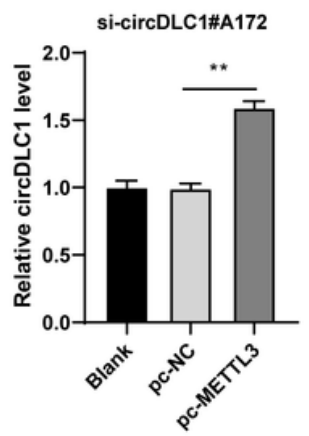

D

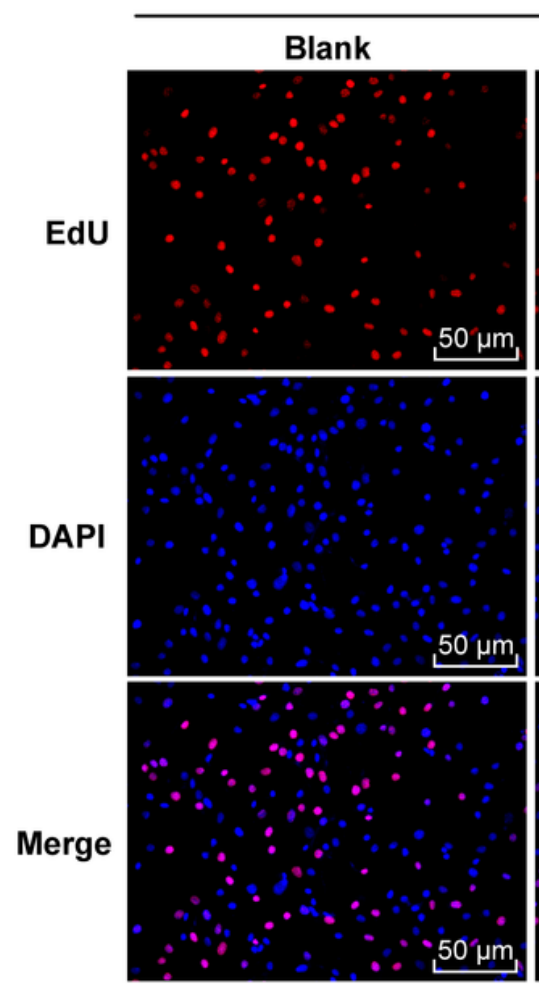

si-circDLC1\#A172

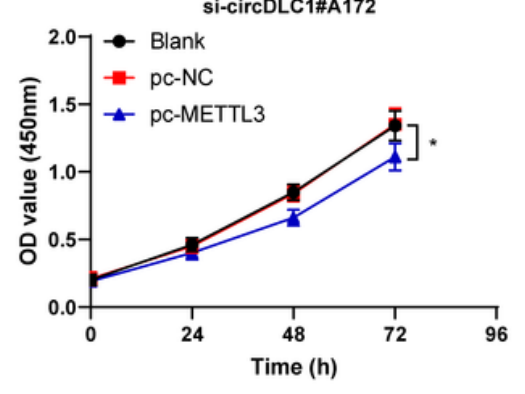

C

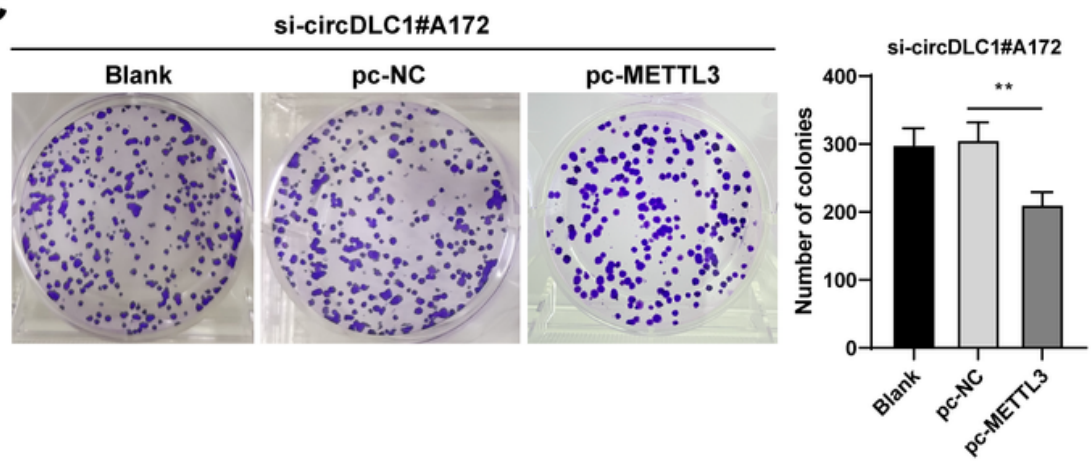

si-circDLC1\#A172

pc-NC
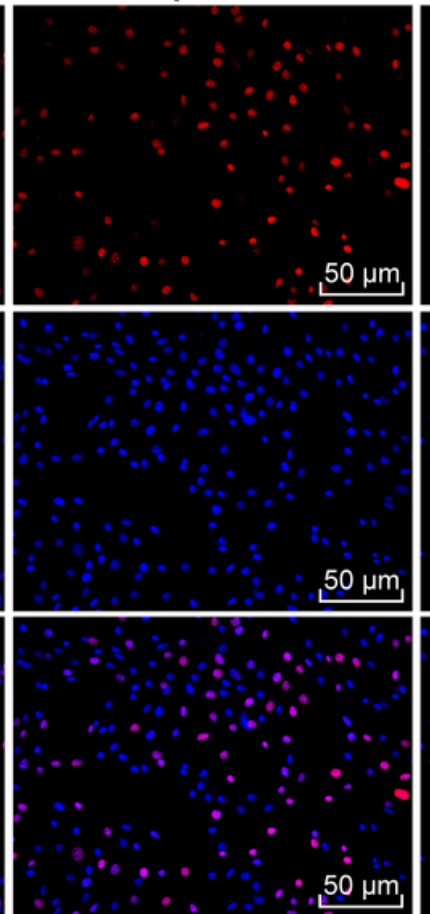

pc-METTL3

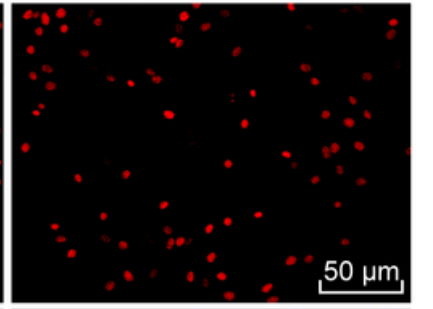

$50 \mu \mathrm{m}$

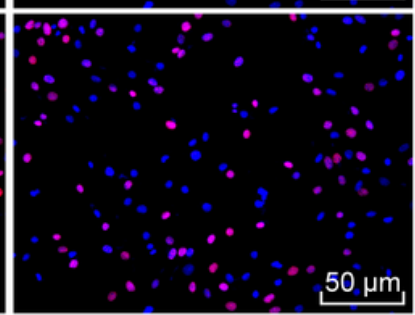

si-circDLC1\#A172

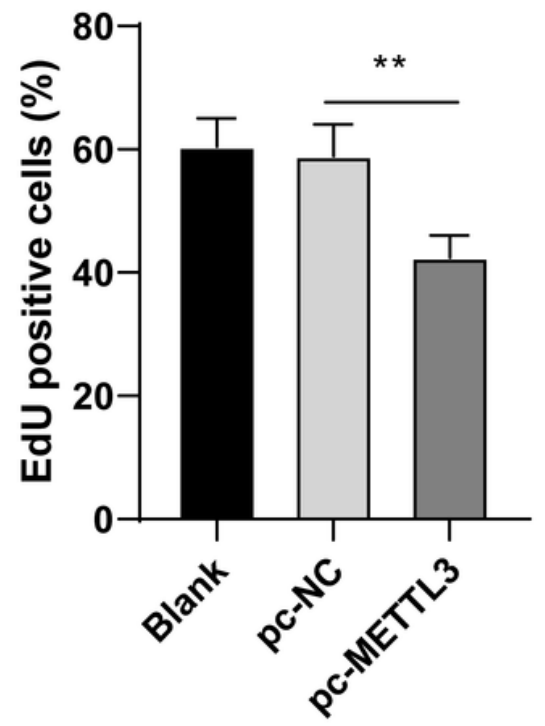

\section{Figure 4}

Overexpression of METTL3 annulled the promoting effect of circDCL1 silencing on malignant proliferation of glioma cells. A172 cells were treated with METTL3 overexpression and circDCL1 silencing. A: circDCL1 expression in cells was detected using RT-qPCR. B-D: Cell proliferation in each group was measured using CCK-8 assay (B), colony formation assay (C), and EdU staining (D). The cell experiment was repeated 3 times independently. Data are presented as mean \pm standard deviation. Data in panels $A / C$-D were analyzed using paired t-test. Data in panel B were analyzed using one-way ANOVA, and data in panel B were analyzed using two-way ANOVA, followed by Tukey's multiple comparisons test, ${ }^{* *} p<0.01$. 
A

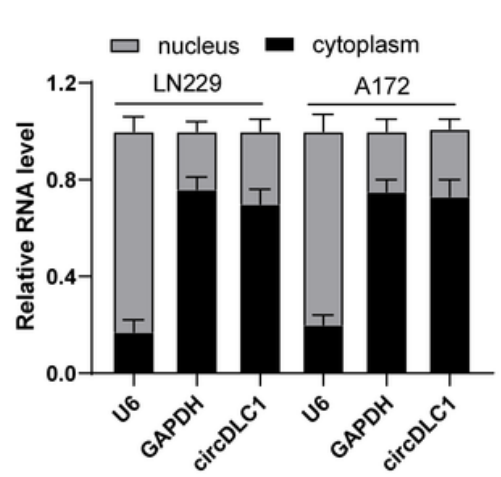

D
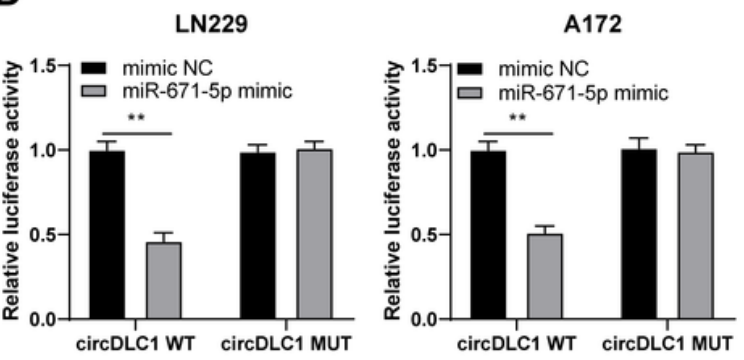

$\mathbf{F}$

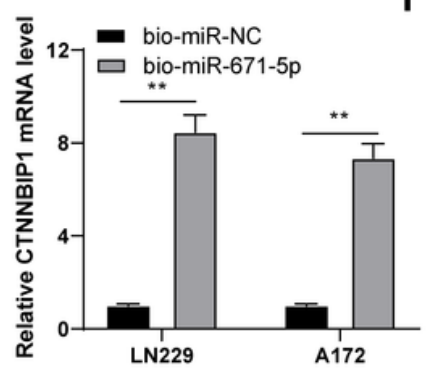

H

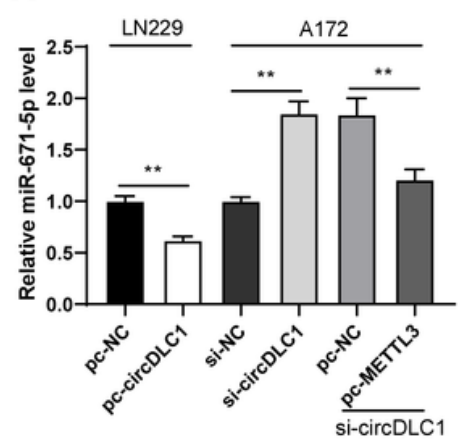

B

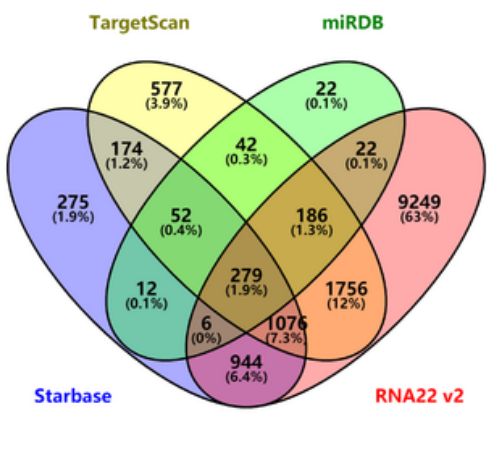

C CircDLC1 3'UTR

miR-671-5p

5'- auauucaa UUUUGAUGGCUUCCa-3'(WT)

$::|:|||||||$

3'- gaggucggGGA GGUCCCGAAGGa -5

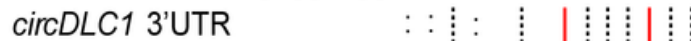

5'- auauucaa UUUUGAUCGCUACCa-3'(MUT)

CTNNBIP1 3'UTR

5'- UGCGCCUCGGUAUCU GGCUUCCC -3' (WT)

miR-671-5p |||||||

3'- GAGGUCGGGGAGGUCCCGAAGGA-5

CTNNBIP1 3'UTR

5'- UGCGCCUCGGUAUCU GGCUAACCC-3'(MUT)

E

A172
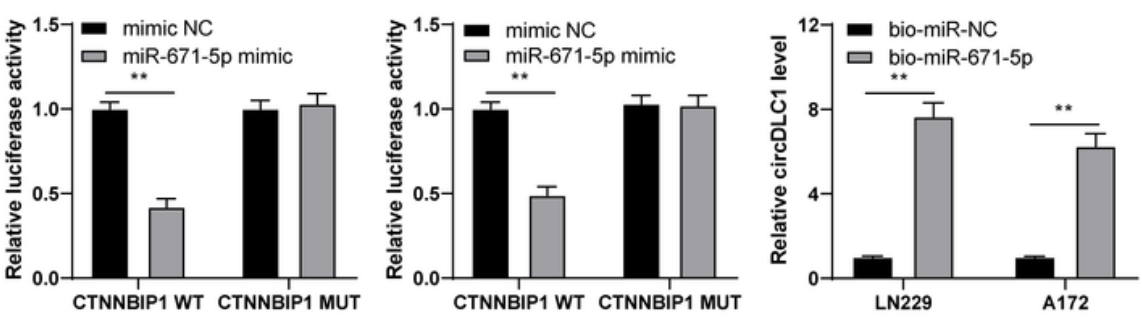

G

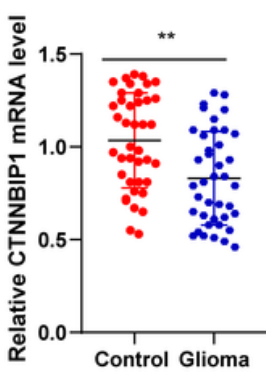

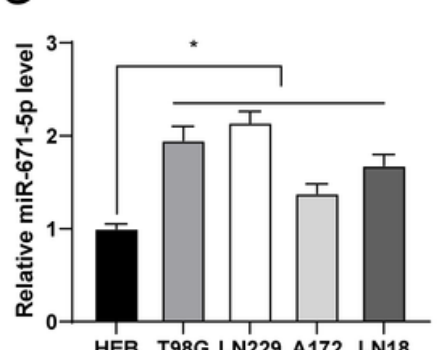

HEB T98G LN229 A172 LN18

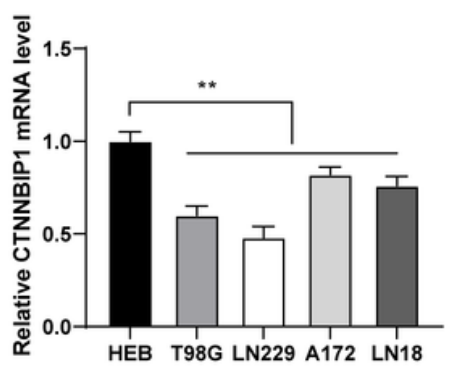

I
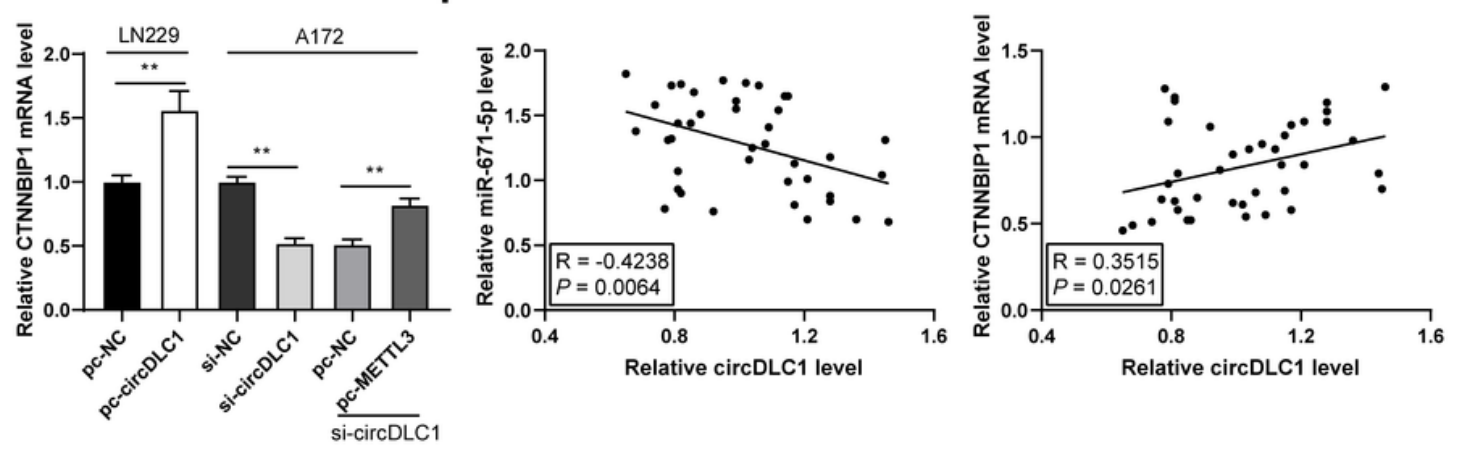

Figure 5

circDCL1 promoted CTNNBIP1 transcription by competitively binding to miR-671-5p. A: The location of circDCL1 in LN229 and A172 cells was analyzed using nuclear/cytosol fractionation assay. B: The downstream target genes of miR-671-5p were predicted through the Starbase, Targetscan, RNA22 v2, and miRDB databases and intersected. C: The binding sites of miR-671-5p with circDCL1 or CTNNBIP1 in the Starbase and Targetscan database. D-E: The binding relationship between miR-671-5p and circDCL1 or CTNNBIP1 was verified using dual-luciferase assay and RNA pull-down assay. F-H: miR-671-5p and 
CTNNBIP1 expressions in glioma tissues and cells were determined using RT-qPCR. I: Pearson correlation analysis of the correlation between miR-671-5p or CTNNBIP1 and circDCL1 in 40 cases of glioma tissues. $\mathrm{N}=40$. The cell experiment was repeated 3 times independently. Data in panels A/D-E/G-H are presented as mean \pm standard deviation. Data in panels $\mathrm{E}-\mathrm{F}$ were analyzed using t-test. Data in panels $\mathrm{G}-\mathrm{H}$ were analyzed using one-way ANOVA, and data in panel D were analyzed using two-way ANOVA, followed by Tukey's multiple comparisons test, ${ }^{*} p<0.05,{ }^{* *} p<0.01$.

A

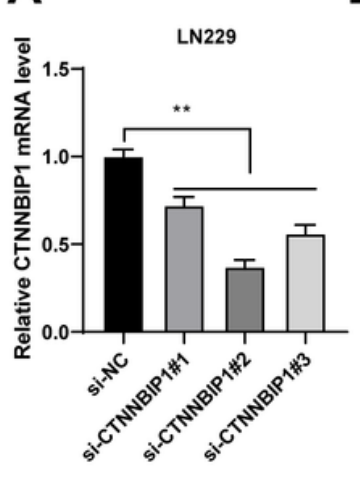

D

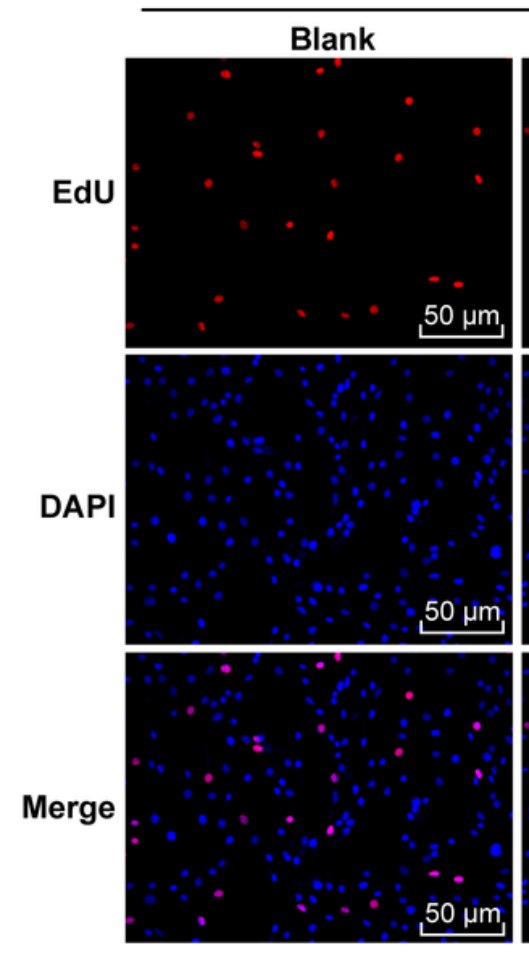

B

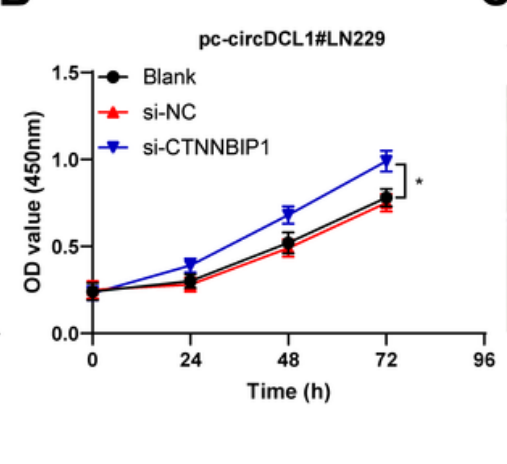

C

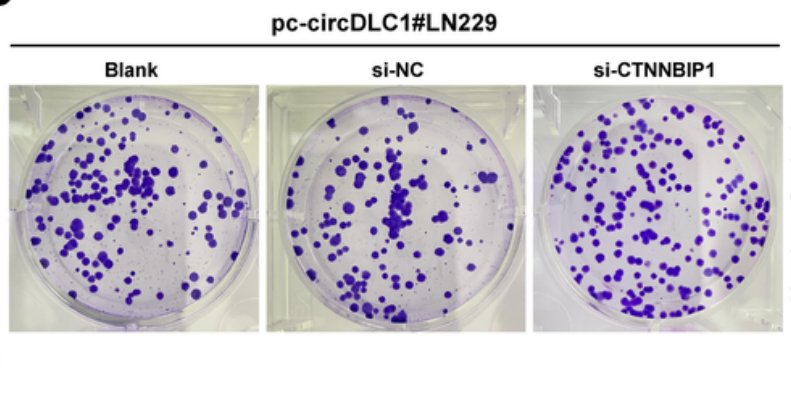

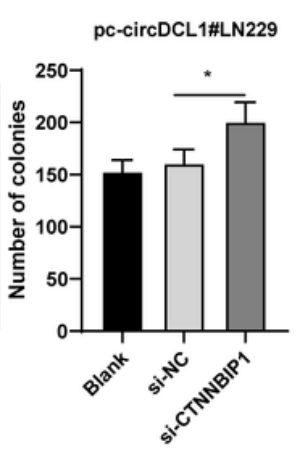

pc-circDLC1\#LN229
si-CTNNBIP1

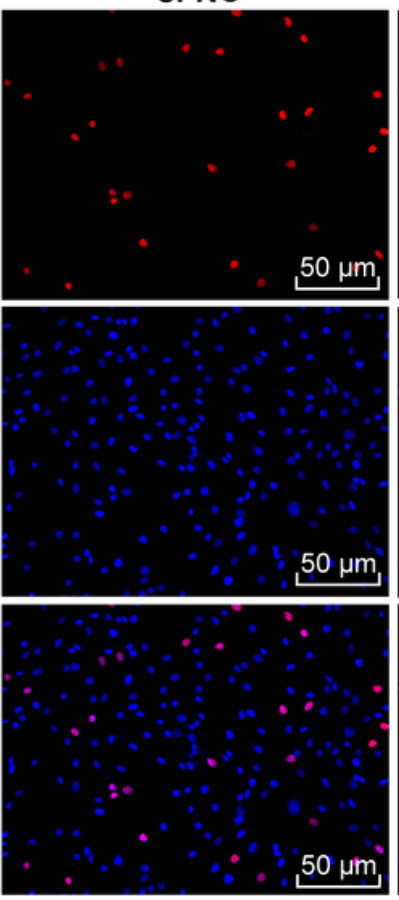

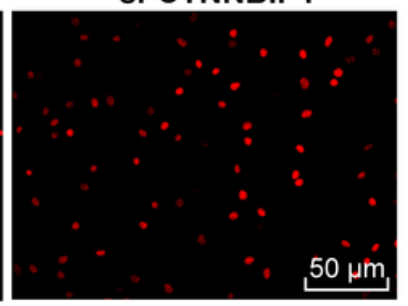
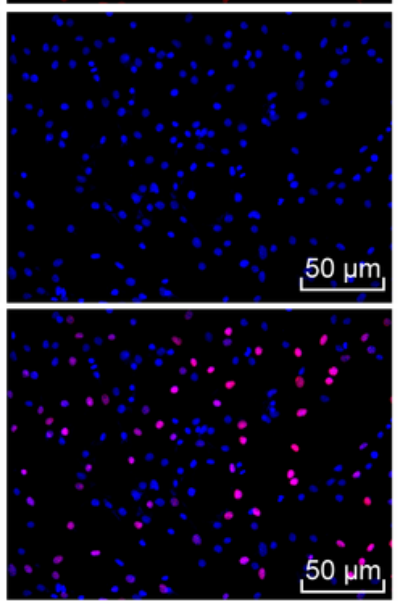

pc-circDCL1\#LN229

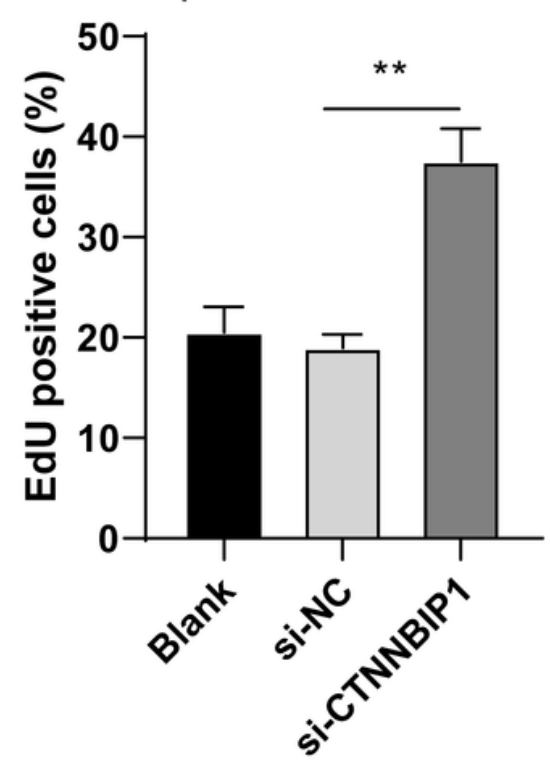

\section{Figure 6}

CTNNBIP1 silencing attenuated the inhibitory effect of circDCL1 overexpression on malignant proliferation of glioma cells. Three CTNNBIP1 siRNAs (si-CTNNBIP1) were transfected into LN229 cells, with NC siRNA (si-NC) as control. A: CTNNBIP1 mRNA expression was determined using RT-qPCR. B-D: Cell proliferation in each group was measured using CCK-8 assay (B), colony formation assay (C), and 
EdU staining (D). The cell experiment was repeated 3 times independently. Data are presented as mean \pm standard deviation. Data in panels A/C-D were analyzed using one-way ANOVA, and data in panel B were analyzed using two-way ANOVA, followed by Tukey's multiple comparisons test, ${ }^{*} p<0.05,{ }^{* \star} p<0.01$.
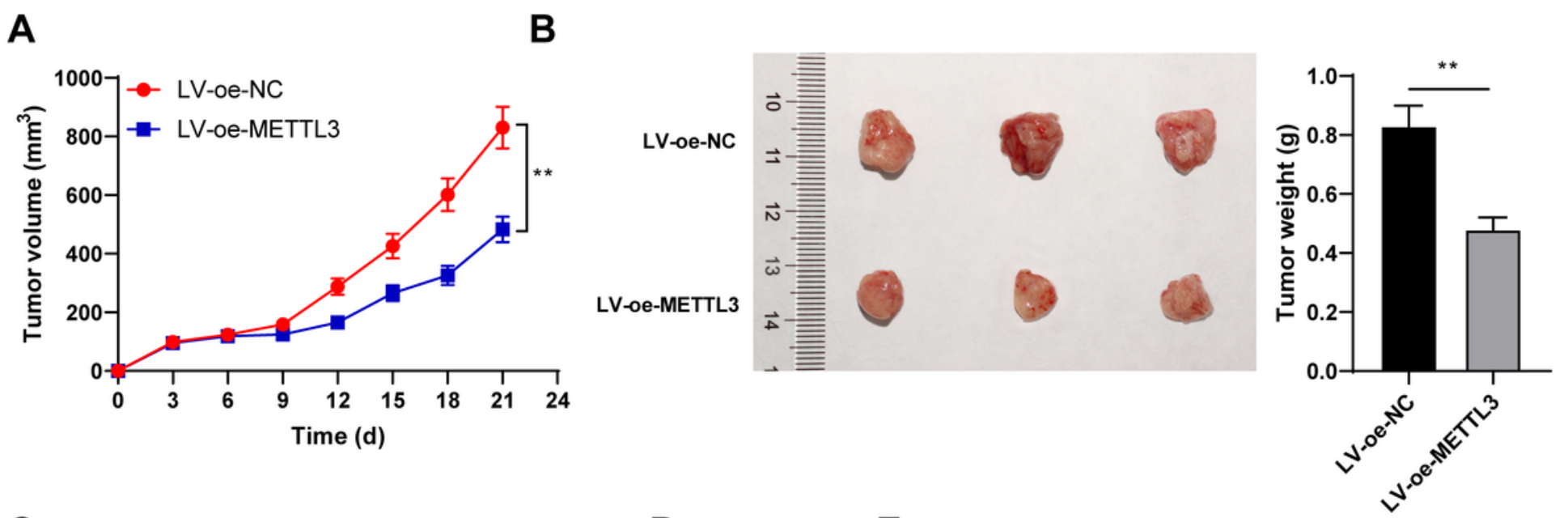

C

D

$\mathbf{E}$
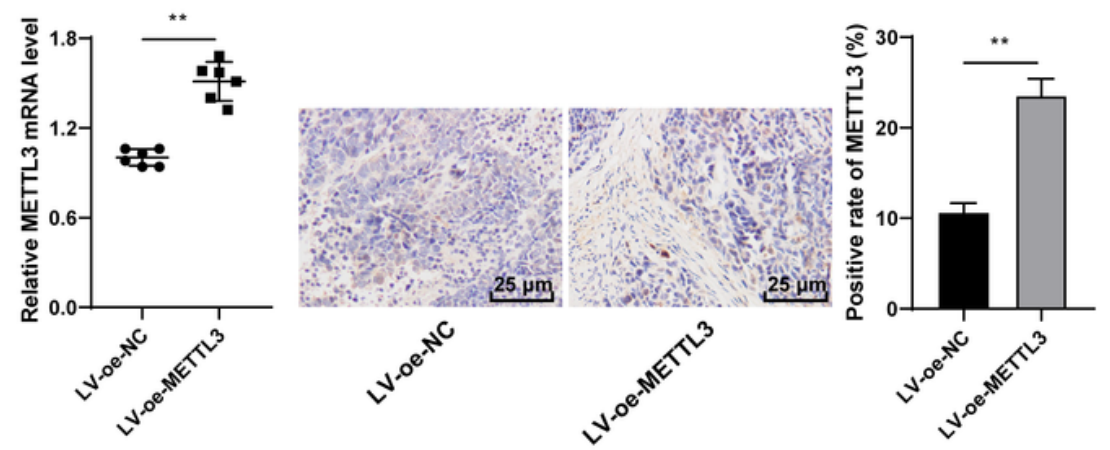

$\mathbf{F}$

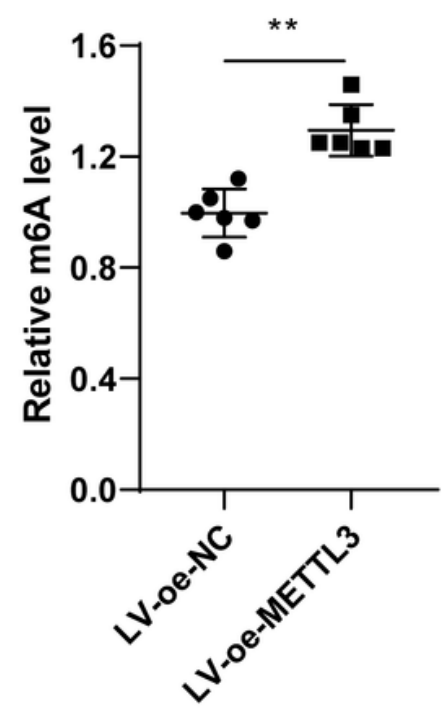

G

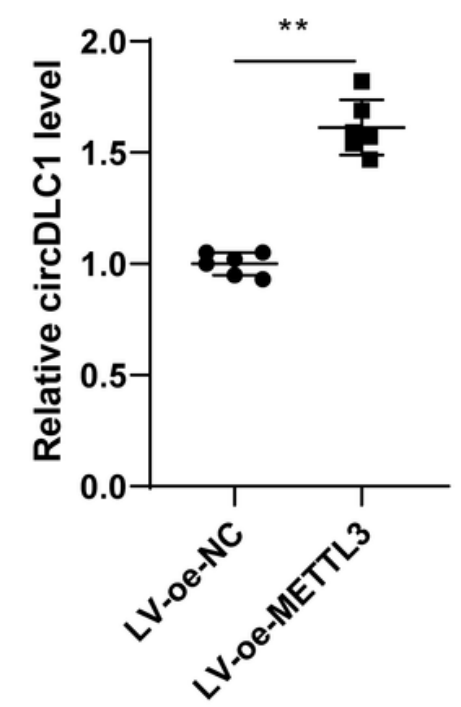

H

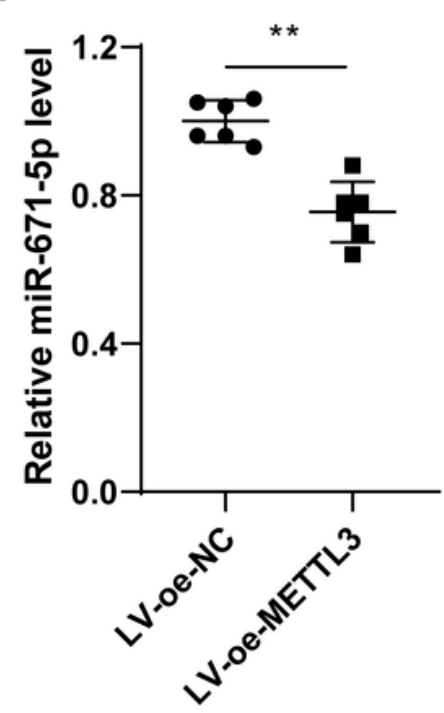

I

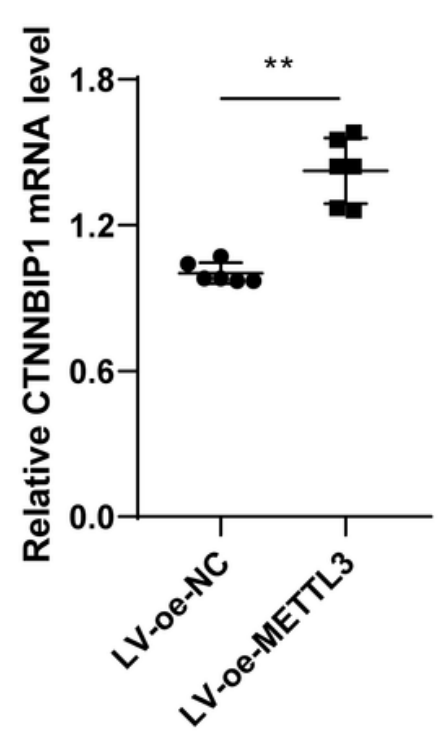

Figure 7 
METTL3 suppressed the malignant proliferation of glioma cells via the circDCL1/miR-671-5p/CTNNBIP1 axis in vivo. LN229 cells stably overexpressing METTL3 (LV-oe-METTL3) were used to establish the xenograft tumor model in nude mice. A: Tumor volume. B: The representative images of the stripped tumors and the weight of the tumors after the nude mice were euthanized on the $21^{\text {st }}$ day. C: Ki67-positive rate in tumor tissues was detected using immunohistochemistry. D-E: METTL3 expression in tumor tissues was determined using RT-qPCR and immunohistochemistry. F: $\mathrm{m}^{6} \mathrm{~A}$ quantitative analysis of $\mathrm{m}^{6} \mathrm{~A}$ level in tumor tissues. G-I: circDCL1, miR-671-5p, and CTNNBIP1 expressions in tumor tissues were determined using RT-qPCR. $N=6$. The cell experiment was repeated 3 times independently. Data in panels $\mathrm{A}-\mathrm{C} / \mathrm{E}$ are presented as mean \pm standard deviation. Data in panels $\mathrm{B}-\mathrm{I}$ were analyzed using t-test. Data in panel A were analyzed using two-way ANOVA, followed by Tukey's multiple comparisons test, ${ }^{* *} p<0.01$.

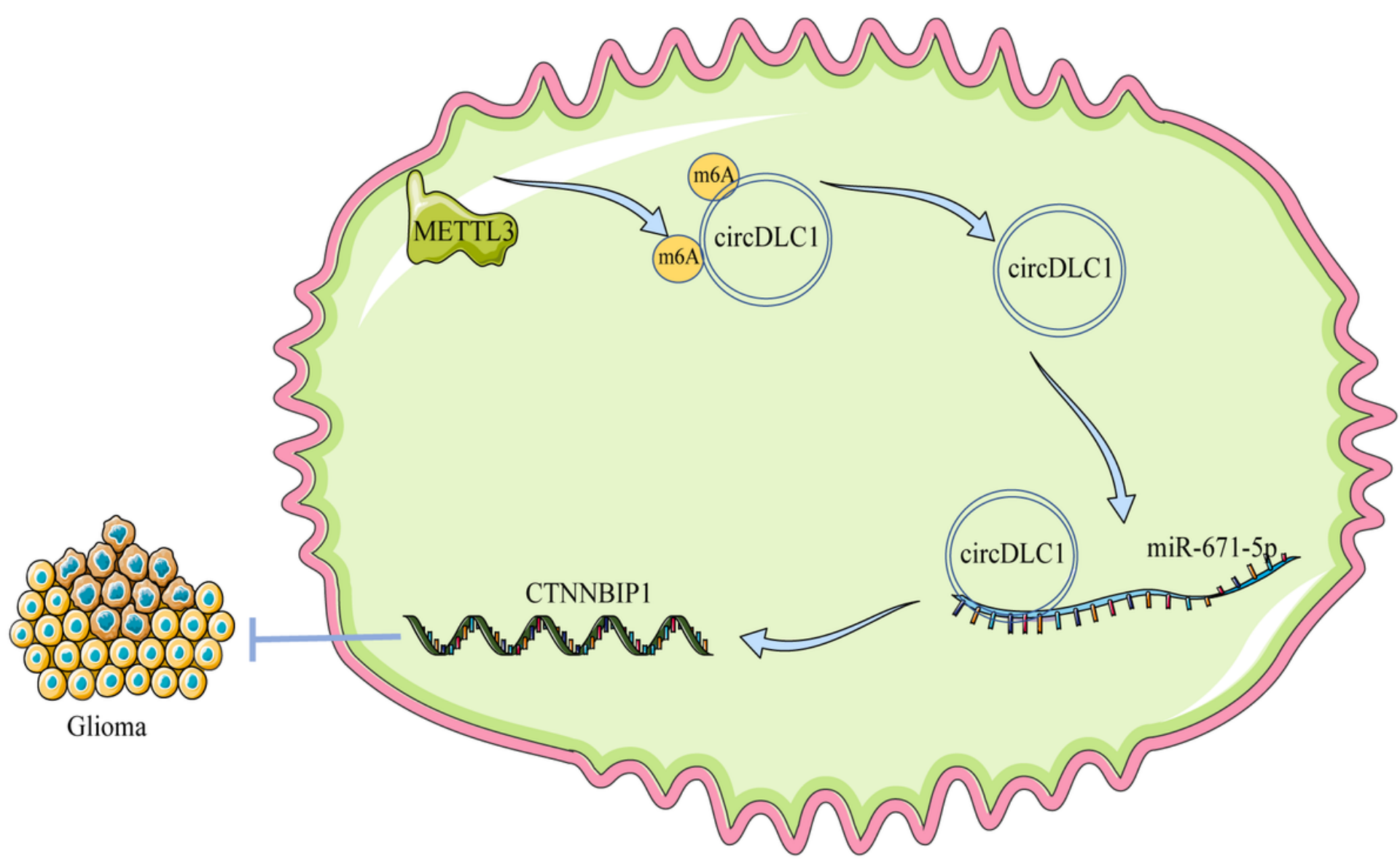

Figure 8

Molecular mechanism of circDCL1 regulating malignant proliferation of glioma cells. METTL3-mediated $\mathrm{m}^{6} \mathrm{~A}$ modification can increase the stability of circDCL1, upregulate the expression of circDCL1 in glioma cells, and promote the competitive binding of circDCL1 and miR-671-5p, thereby upregulating the transcription of CTNNBIP1 and then inhibiting the malignant proliferation of glioma cells. 
Page 27/27 